\title{
An analytical model of asymmetric rolling of unbounded clad sheets with shear effects
}

\author{
F. Afrouz ${ }^{a}$, A. Parvizi ${ }^{b}$
}

${ }^{a}$ M.Sc. Student, Department of Mechanical and Aerospace Engineering, Science and Research Branch, Islamic Azad University, Tehran, Iran

${ }^{\mathrm{b}}$ Corresponding Author, Assistant Professor, School of Mechanical Engineering, College of Engineering, University of Tehran, Tehran, Iran

Address: North Kargar St, School of Mechanical Engineering, University Of Tehran College of Engineering, Tehran, Iran

E-mail: aliparvizi@ut.ac.ir, Tel: +98 2182084026 
Abstract: Based on the slab method analysis, anew solution for asymmetrical rolling of unbonded clad sheet is presentedin this paper. The non-uniformity of the shear stresses and the uniformity of the normal stresses at the vertical sides of each slab across the portion of the deforming material all over the plastic region are considered. The effects of the process parameters such as reduction, work rolls radii ratio and work rolls speed ratio on process outputs are investigated.In addition, the asymmetrical clad sheet unbonded before rolling processis simulated wholly using ABAQUS/explicit software. Comparisons of analytical results from present model with those from experimental studies of the other investigators and the finite element simulation have showed good agreements.

Keywords: Asymmetrical rolling;clad sheet; Pressure distribution; Slab method; FEM. 


\section{Nomenclature}

F $\quad$ Rolling force of the asymmetrical clad sheet rolling

$F_{1}, F_{2}, F_{3}, \quad$ Rolling forces in zone $I, I I, I I I$ and $I V$,respectively

$F_{4}$

$h$

Variable sheet thickness at the roll gap

$h_{1}, h_{2} \quad$ Upper and lower element heights with respect to horizontal axis, respectively

$h_{i}, h_{o} \quad$ Sheet thicknesses at the entry and the exit of the roll gap, respectively

$m_{1}, m_{2} \quad$ Friction factors at the upper and the lower rolls respectively

$m_{3} \quad$ Friction factor at the interface of the clad sheet

$k_{1}, k_{2} \quad$ Mean yield shear stresses of the upper and the lower sheets, respectively

$L \quad$ Contact length

$p \quad$ Vertical stress at the roll gap

$p_{1}, \quad p_{2} \quad$ Rolling pressures at the upper and the lower interfaces, respectively

$p_{3} \quad$ Contact pressure between the clad sheet

$r$

Reduction in thickness (\%)

$\mathrm{R}_{1}, \mathrm{R}_{2} \quad$ Radii of the upper and the lower work rolls, respectively

$R_{e q} \quad$ Equivalent work roll radius

$T \quad$ Total rolling torque of the asymmetrical clad sheet rolling

$T_{1}, T_{2} \quad$ Rolling torques of the upper and the lower rolls, respectively

$V_{A} \quad$ Ratio of the lower to the upper work roll speeds

$x_{n 1}, \quad x_{n 2}$ Positions of the upper and the lower neutral points, respectively 
$x_{b} \quad$ Position of the bonding point

$\sigma_{1}, \quad \sigma_{2} \quad$ Normal stresses at the upper and the lower slab element in the roll gap

$\tau_{1}, \tau_{2} \quad$ Frictional shear stresses at the upper and the lower interfaces, respectively

$\tau_{3} \quad$ Shear stress at the interface of the clad sheet

$\bar{\tau}_{1}, \quad \bar{\tau}_{2} \quad$ Average shear stresses along the vertical side of the upper and the lower slab element, respectively

$\theta_{1}, \theta_{2} \quad$ Variable angles of contact for the upper and the lower rolls, respectively

$\sigma_{y p 1}, \quad$ Flow stresses of the upper and the lower sheets, respectively

$\sigma_{y p 2}$ 


\section{Introduction}

Due to some unique features such as high strength, high resistivity against corrosion, and high electrical conductivity, the usages of laminated sheets have beenrisen. There are many different methods for mass production of laminated sheets in which the asymmetrical rolling is one of the efficientprocesses. In the asymmetrical clad sheet rolling, the work rolls radii, speeds, surface roughness and thickness of the sheets may be different. Compared to symmetrical process, the rolling force, pressure, and torque can be decreased in the asymmetrical process and the energy can be saved.

Up to now, a number of studies have been done in the field of the asymmetrical sheet and clad sheet rolling processes. A mathematical model for analysis of the asymmetrical clad sheet rolling process based on the stream function and the upper bound methods were done by Hwang and Chen [1]. Lee and Lee [2] used the slab method and experimental analysis to study roll bonding of silver clad phosphor bronze sheets. Some experimental studies were done by Johnson and Needham [3] to investigate the asymmetrical sheet rolling.

Finite element method (FEM) analysis is one of the powerful methods to determine the stress-strain distribution in the metal forming processes. But, a lot of time and cost are required to carry out this method. FEM was used to evaluate the effects of shear yield stress ratio and thickness ratio on the stress, strain, and rolling force in clad sheet rolling process [4-5]. 
The slab method is a proper method can be used to analyze metal forming processes like symmetrical and asymmetrical sheet and clad sheet rolling. Via this method, the rolling force and torque can be predicted rapidly and a large amount of solution time can be saved. Studies on the asymmetrical rolling process can be categorized into two groups, the asymmetrical sheet rolling and the asymmetrical clad sheet rolling.

Althoughsomeresearchers have used the slab method to investigate the asymmetrical sheet and clad sheet rolling processes, but this method with considering the nonuniform distribution of shear stress acting on a vertical slab across the section of the deforming material has never been applied to clad sheet rolling process.

An analyticalmodel for analysis of relationship between frictional coefficient and frictional factor in asymmetrical sheet rolling was proposed by Tzou [6]. Based on that analysis, the frictional factor can be obtained rapidly and easily when the frictional coefficient is known. In author's previous study [7], an analytical solution for ring rolling process was presented according to the slab method theory in which the non-uniformity of the normal and shear stresses across the section of the deforming material throughout the plastic zone was considered. Taking into consideration the strainhardening effects,Gudur et al. [8] attempted to analyze the asymmetrical rolling process in order to estimate the curvature of the rolled sheet. Using the slab method, the analysis of the asymmetrical sheet rolling process wascarried out by Yong et al. [9]. They investigated the influence of asymmetrical rolling factors on deformation area and rolling pressure by dividing the deformation area into three zones. An analyticalsolution was proposed by Tzou and Huang [10] to investigate the minimum thickness for the asymmetrical PV cold rolling of sheet.They considered two analytical models with the 
same and the different frictional coefficients at the upper and lower rolls. In addition, another model was proposed by Tzou and Huang [11]to analyze the minimum thickness for asymmetrical hot-and-cold PV rolling by considering the constant shear friction.

Based on the slab method theory, an analytical solution was presented by Hwang and Tzou [12]for asymmetrical sheet rolling process in which the effects of the shear stress and internal moment at the roll gap were considered. Gao et al. [13] studied the asymmetrical cold rolling by considering the differences in the coefficient of friction between rolls and sheets rather than the differences of rolls radii or rotation speeds. According to the slab method, Salimi and Kadkhodaei [14] considered non-uniformity of the normal and shear stresses in deriving the governing equations for analysis of plane strain asymmetrical rolling. Likewise, Zhang et al. [15] presented a new stress field for asymmetrical sheet rolling in which the uniform normal and non-uniformshear stresses on the vertical sides of each slab were considered.In the earlier works on clad sheet rolling, the slab method mainly was applied to evaluate the rolling pressure and force. Furthermore, the slab method was also applied to investigate the plastic deformation of clad sheet, considering constant shear friction and hybrid friction, respectivelyin [16-17]. An analytical model was presented by Pan et al.[18]for analysis of asymmetrical cold and hot bond rolling of unbonded clad sheet. In their model,they assumed uniform normal stress, but ignored the shear stress distribution in the vertical sides of the slabs.However, the physical condition of the asymmetricalrolling of unbounded clad sheets in which different friction states exist at the interfaces of clad sheet with the upper and the lower rolls and at the interface of two layers necessitate to consider a non-uniform distribution of the shear stress on the vertical sides of the slabs. Therefore, 
this postulate is taken into account in this study as a novelty to extend the Pan et al. [18] study and obtain a more accurate model.

In this paper, a solution based on the slab method of analysis is presented for asymmetrical clad sheet rolling process. At first, a mathematical model for the stress field acting on a vertical slab in the roll gap is presented in which the uniformity of the normal stress and non-uniformity of shear stress across the section of the deforming material are considered. After that, imposing the force balance on the slab with differential thickness, one dimensional differential equation for pressure at the roll gap is obtained. Finally, imposing relevant boundary conditions on the differential equation provides an analytical solution for the rolling pressure. In the analytical solution, a variety of factors for asymmetrical clad sheet rolling process such as the friction factor, the friction factor ratio, the roll speed ratio, and the roll radii ratio have been used to investigate the behavior of the process. Based on the present analysis, complete expressions for the asymmetrical clad sheet rolling pressure, force and torque are obtained and the position of the bonding point and neutral points are determined. Eventually, by using ABAQUS/explicit software, the asymmetrical clad sheet rolling process is simulated and the results are used to evaluate the accuracy of present model.

\section{Mathematical models}

In Fig.1, the schematic illustration of the asymmetrical clad sheet rolling unbonded before rolling is shown.According to this figure, two unbonded metal sheets with uniform unit thickness and a total heights $h_{i}$ and $h_{o}$ at the entry and the exit,respectively are 
rolled through the asymmetrical rollers. The upper sheet is softer than the lower one and has lower yield stress. The horizontal distance from the exit point in the roll gap is taken as the $\mathrm{x}$-axis and the origin is its intersection with the normal plane passing by centers of two rolls.

The plastic deformation zone at the roll gap can be divided into four different zones. Zone I for the unbonded zone $\left(x_{b} \leq x \leq L\right)$ in which only the softer sheet,the upper layer, is yielded.When the harder sheet is alsoyielded,the bonding point $\left(x_{b}\right)$ is generatedand after that, the sheets are bonded together completely. In zone II $\left(x_{n 1} \leq x \leq x_{b}\right)$, the peripheral velocities of the upper and the lower rolls are larger than that of the sheets, thus the direction of both the upper and lower frictions on the clad sheet are in the forward direction. Zone III $\left(\mathrm{x}_{\mathrm{n} 2} \leq \mathrm{x} \leq \mathrm{x}_{\mathrm{n} 1}\right)$ is a cross shear zonein which the clad sheet velocity is lessthan that of the lower roll and greater than that of the upper roll.Hence, the frictional stress on the upper surface (between the upper roll and the clad sheet) is backward and on the lower surface (between the lower roll and the clad sheet) is forward. In zone $I V\left(0 \leq \mathrm{x} \leq \mathrm{x}_{\mathrm{n} 2}\right)$, the velocity of the clad sheet is higher than the peripheral velocities of the upper and lower rolls and the frictional stress on both sides of the clad sheet are in backward direction. A neutral point on the upper roll $\left(x_{n 1}\right)$ is the point at which the peripheral speed of the upper roll becomes equal to the clad sheet velocity.Moreover,the point atwhich the peripheral speed of the lower roll gets equal to the velocity of the clad sheet is the location of the neutral point on the lower roll $\left(x_{n 2}\right)$. 


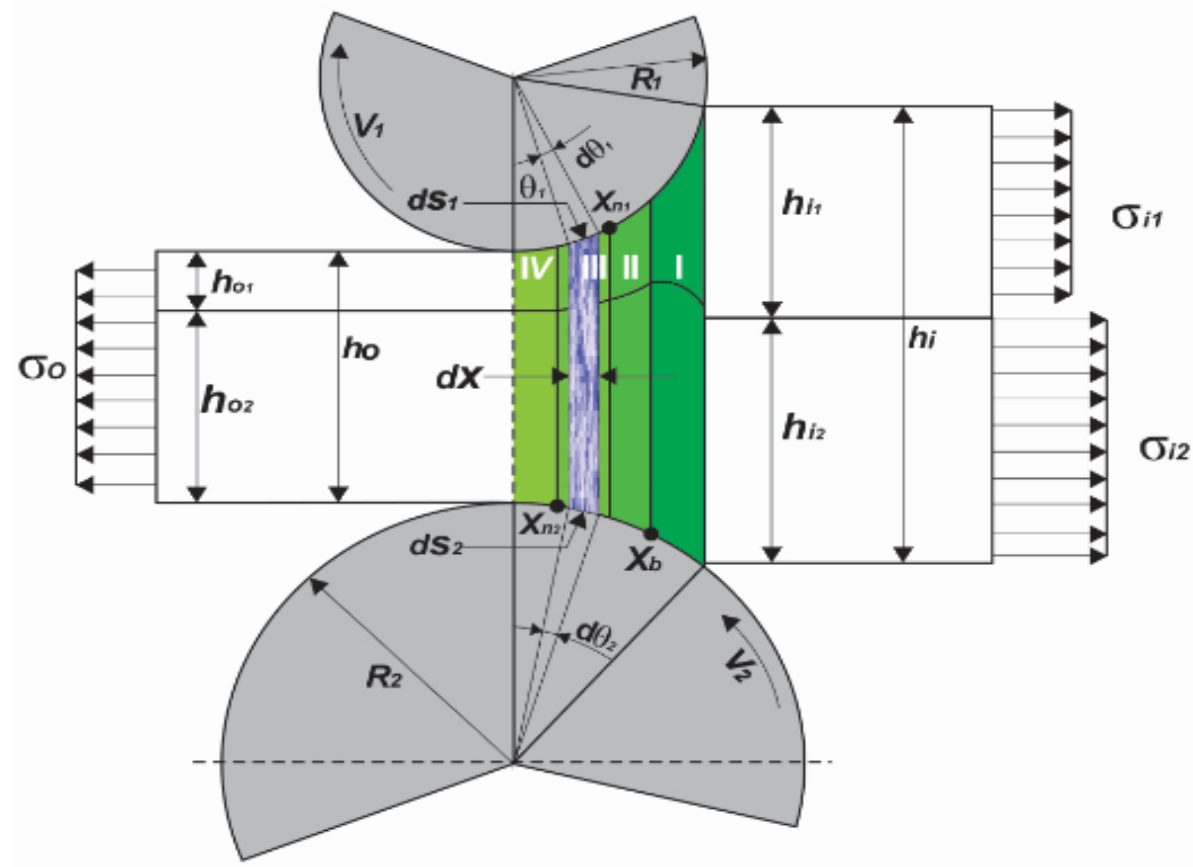

Fig.1 Schematic illustration of the asymmetrical clad sheet rolling process

In the development of the mathematical model, it was assumed that the rolls are rigid and the material being rolled is rigid perfectly plastic. The plastic deformation occurs under plane strain condition, so material spread in the transverse direction is ignored. The condition of constant shear friction is assumed throughout the arc of interaction, i.e. $\tau=m k$. As simulating hot rolling, friction stress $\tau$ is equal to the mean shear yield strength $\mathrm{k}$, namely $m=1$. In passing through the roll gap, the clad sheet thickness is steadily reduced and the clad sheet length and velocity progressively increased from entry to exit.Thespeeds of rolls surfaces have some intermediate values between these entry and exit speeds. The contact length is small as compared with the roll circumference and can be obtained from the following [1] $L=\sqrt{R_{e q} \cdot h_{i} \cdot r}$ 
Where $R_{e q}$ is equivalent work roll radiusand can be obtained from Eq 2, $r$ is the reduction in thickness and $h_{i}$ is the total sheet thickness at entry.Subscripts 1 and 2 are designated to quantities at the upper and the lower sheetsand rolls, respectively. From simple geometry

$$
\begin{gathered}
h_{1}=\frac{h_{o}}{2}+\frac{x^{2}}{2 R_{1}}, h_{2}=\frac{h_{o}}{2}+\frac{x^{2}}{2 R_{2}}, h=h_{1}+h_{i 2}=h_{o}+\frac{x^{2}}{R_{e q}}, R_{e q}=\frac{2 R_{1} R_{2}}{\left(R_{1}+R_{2}\right)} \\
\tan \theta_{1}=\frac{x}{R_{1}}, \tan \theta_{2}=\frac{x}{R_{2}}, \frac{d h}{d x}=\frac{2 x}{R_{e q}}
\end{gathered}
$$

Where $h$ is a variable sheet thickness at the roll gap; $h_{1}$ and $h_{2}$ are upper and lower element heights with respect to horizontal axis, respectively; $\theta_{1}$ and $\theta_{2}$ are variable contact angles; $R_{1}$ and $R_{2}$ denote the radii of the upper and lower rolls, respectively.

The equivalent stress fieldsacting on vertical elements at zones I,II,III and IVare shown in Fig.2. This figure includes the uniformity of the normal stresses in all vertical elements. But, the shear stresses on the vertical sides of the differential elements vary from $\tau_{1}=m_{1} k_{1}$ at the upper surface to $\tau_{2}=m_{2} k_{2}$ at the lower surface. Forthe upper sheet in zonesIand $I I$ and the lower sheet in zone $I V$, the shear stresses obtain their minimum values zero at points in between the vertical side and the shear stresses are linearly distributed from the upper and lower surfaces to thesepoints. But, in zones $I I I$ and $I V$ of the upper sheet and zones I,IIand IIIof the lower sheet, the shear stresses are linearly distributed from the upper surface to the lower surface and at the center, itbecomes equal to mean value of its surfaces. 

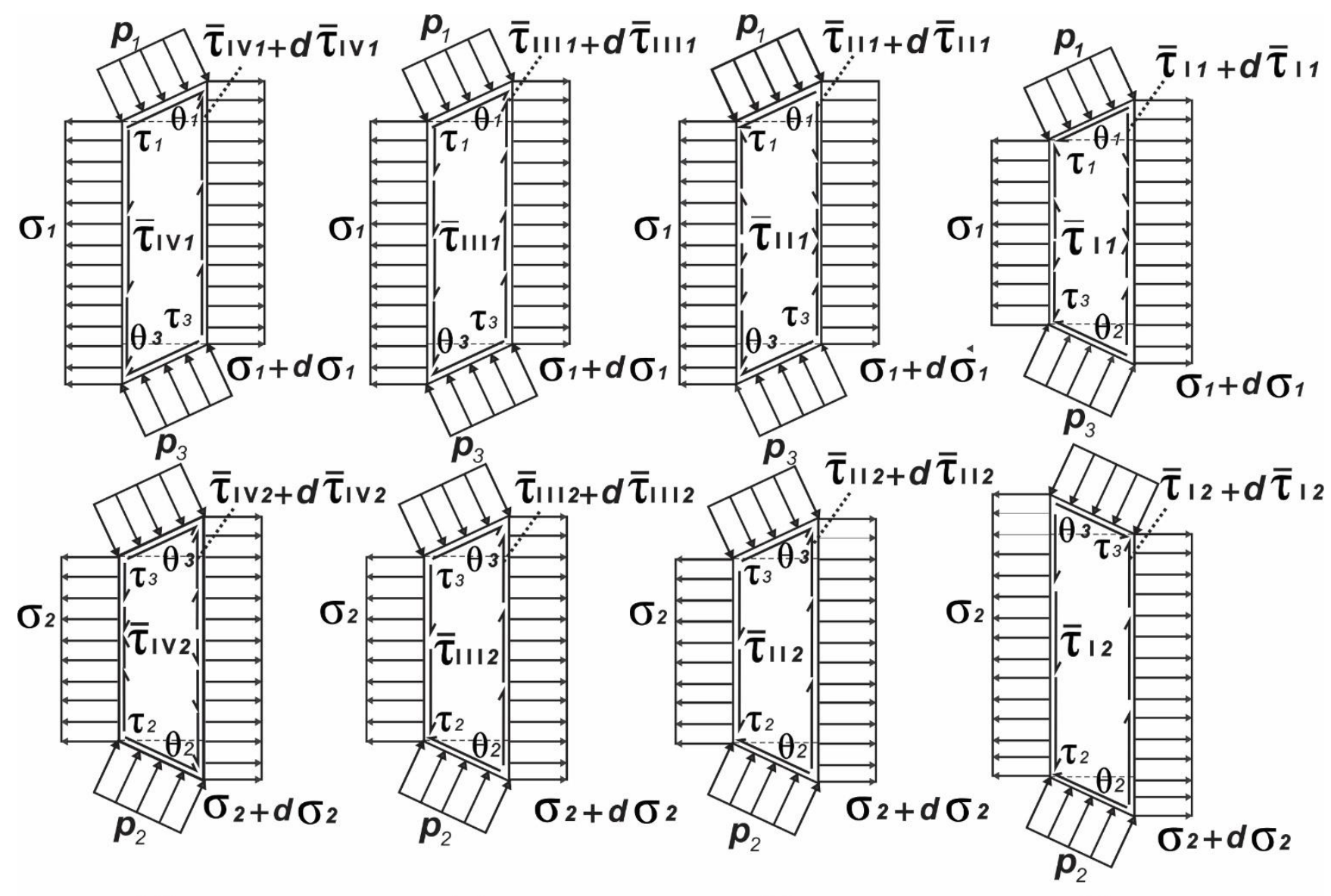

(d)

(c)

(b)

(a)

Fig. 2 Stress fields acting on a vertical elements at (a) zoneI (b) zoneII (c) zoneIII (d) zoneIV

\subsection{Clad sheet rolling pressure}

\subsubsection{Zone $I\left(x_{b} \leq x \leq L\right)$}

In zone $I$, the harder sheet (layer2) is not yielded which means; $p+\sigma_{2}<2 k_{2}$, but the softer sheet (layer1) is yielded with the yield criterion; $p+\sigma_{1}=2 k_{1}$ where $k_{1}=\frac{\sigma_{y p} 1}{\sqrt{3}}$, $k_{2}=\frac{\sigma_{y p}}{\sqrt{3}} \cdot \sigma_{y p 1}$ and $\sigma_{y p 2}$ are the mean yield stresses of the upper and lower sheets, respectively. 
Based on the stress field of Fig. 2 (a), the horizontal and vertical force equilibriums for the softer sheet lead to the following relations;

$\frac{d\left(h_{1} \sigma_{1}\right)}{d x}+p_{1} \tan \theta_{1}-p_{3} \tan \theta_{3}-\left(\tau_{1}+\tau_{3}\right)=0$

$p=p_{1}+\tau_{1}-\tau_{3} \frac{d\left(h_{1}\right)}{d x}=p_{3}-\tau_{3} \tan \theta_{3}$

where $\sigma$ and pdenote the horizontal and the vertical stresses, respectively; $\tau_{3}$ and $\theta_{3}$ are the shear stress and the contact angle at the interface of the clad sheet, respectively.Since the harder sheet has not yielded, the contact angle $\theta_{3}$ at the interface becomes equalto the contact angle $\theta_{2}$ between the roll and clad sheet in zoneI[18]. While the directions of both the upper and the lower frictions on the clad sheet are forward, the equivalent shear stress at zone $I$ is defined as $\tau_{e 1}=m_{1} k_{1}+m_{3} k_{1}$. The distributions of shear stress on the vertical elements in zone $I$, for the upper layer is shown in Fig. 3. According to Fig. 3, the shear stresses are equal to $\tau_{1}$ and $\tau_{3}$ at the upper and lower interfaces of the upper sheet, respectively and distributed linearly towards the both sides.

Combining Eqs 3 with 4 and using the Von-Mises yield criterion as well as the geometrical and frictional conditions, the following relation is obtained

$\frac{d p}{d x}=\frac{4 k_{1}}{\left(x^{2}+R_{e q}\left(h_{o}-h_{i 2}\right)\right)} x-\frac{S_{1}}{\left(x^{2}+R_{e q}\left(h_{o}-h_{i 2}\right)\right)} x^{2}-\frac{R_{e q} \tau_{e 1}}{\left(x^{2}+R_{e q}\left(h_{o}-h_{i 2}\right)\right)}$

Solving the resulted differential equation, the rolling pressure distribution in zone $I, p_{\mathrm{I}}$, can be obtained as follows

$p_{I}=-S_{1} x+\frac{H_{1}}{2} \ln \left(x^{2}+U_{1}\right)+C_{d 1} \omega_{1}+B_{1}^{*}$ 


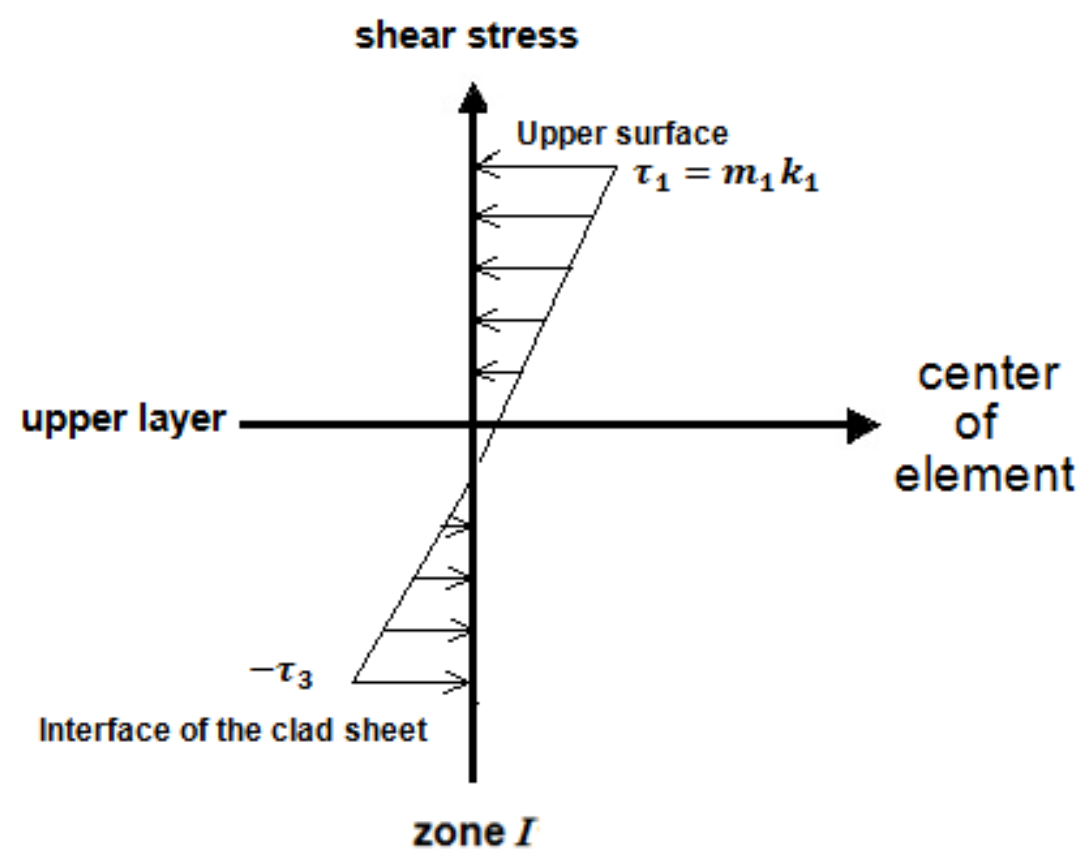

Fig. 3. Distribution the shear stress on the vertical elements of zone $I$ for the upper layer

where $B_{1}^{*}$ is a constant of integration which will be derived later and

$S_{1}=\left(\frac{\tau_{1}}{R_{1}^{2}}+\frac{\tau_{3}}{R_{2}^{2}}+\frac{2 \bar{\tau}_{I 1}}{R_{1} R_{e q}}\right), \quad H_{1}=4 k_{1}, \quad U_{1}=R_{e q}\left(h_{o}-h_{i 2}\right)$

$L_{1}=\tau_{e 1} R_{e q} \quad, \quad C_{d 1}=\left(\frac{S_{1} U_{1}-L_{1}}{\sqrt{U_{1}}}\right), \quad \omega_{1}=\tan ^{-1} \frac{x}{\sqrt{U_{1}}}$

$\bar{\tau}_{I 1}=\frac{1}{4}\left(\tau_{1}-\tau_{3}\right)$

Since the softer sheet is yielded, $\tau_{3}=m_{3} k_{1}$, and $k_{1}$ is the mean yield shear stress of the softer sheet.In view of the fact that the normal stress at entry side of the roll gap is equal to $\sigma_{i 1}$, the boundary condition at the entry is

$$
\sigma_{1}=\sigma_{i 1} \quad \text { or } p_{i}=2 k_{1}-\sigma_{i 1} \quad \text { at } \quad x=L\left(\text { or } \omega=\omega_{i 1}\right)
$$

Applying Eq 8into 6, the constantB $B_{1}^{*}$ can be obtained as follows 


$$
B_{1}^{*}=2 k_{1}-\sigma_{i 1}-\left(-S_{1} L+\frac{H_{1}}{2} \ln \left(L^{2}+U_{1}\right)+C_{d} \omega_{i 1}\right)
$$

Given that the harder sheet in zone Ihas not yielded, itsthickness does not change, i.e. $h_{2}=h_{i 2}$ and $\frac{d h_{2}}{d x}=0$. Based on the stress field of Fig. 2 (a), the horizontal and vertical force equilibriums for the harder sheet lead to the following relation

$\frac{d \sigma_{2}}{d x}=\frac{\tau_{2}-\tau_{3}}{h_{i 2}}\left(\frac{x^{2}}{R_{2}^{2}}+1\right)$

Solving the differential Eq10, the horizontal stress of layer 2 in zone $I, \sigma_{2}$ can be obtained as follows

$\sigma_{2}=A_{1} x^{3}+D_{1} x+B_{q}^{*}$

where

$A_{1}=\frac{D_{1}}{3 R_{2}^{2}} \quad, \quad D_{1}=\frac{\tau_{2}-\tau_{3}}{h_{i 2}}$

And $B_{q}^{*}$ is a constant of integration which can be derived using the boundary condition $\sigma_{2}=\sigma_{i 2}$ at $x=L\left(\right.$ or $\left.\omega=\omega_{i 1}\right)$ as follows

$B_{q}^{*}=\sigma_{i 2}-\left(A_{1} L^{3}+D_{1} L\right)$

When the harder sheet starts to yield at $x_{b}$, the yield criterion for this layer becomes $p+$ $\sigma_{2}=2 k_{2}$. Hence, the bonding point $\left(x_{b}\right)$ can be obtained from the following equation

$\left(-S_{1} \mathrm{x}_{\mathrm{b}}+\frac{H_{1}}{2} \ln \left(\mathrm{x}_{\mathrm{b}}^{2}+U_{1}\right)+C_{d 1} \omega_{b 1}+B_{1}^{*}\right)+\left(A_{1} x_{b}{ }^{3}+D_{1} x_{b}+B_{q}^{*}\right)=2 \mathrm{k}_{2}$

where

$$
\omega_{b}=\tan ^{-1} \frac{x_{b}}{\sqrt{U_{1}}}
$$

$\mathrm{x}_{\mathrm{b}}$ can easily be obtained by numerical solution. 


\subsubsection{Zone $I I\left(x_{n 1} \leq x \leq x_{b}\right)$}

In zone II, the velocities of the upper and the lower rolls are larger than the velocity of the sheet, thus the direction of both the upper and lower frictions on the clad sheet in zone II are forward i.e. $\tau_{e 2}=m_{1} k_{1}+m_{2} k_{2}$. In Fig. 4 , the shear stress distributions on the vertical elements in zone II are shown.

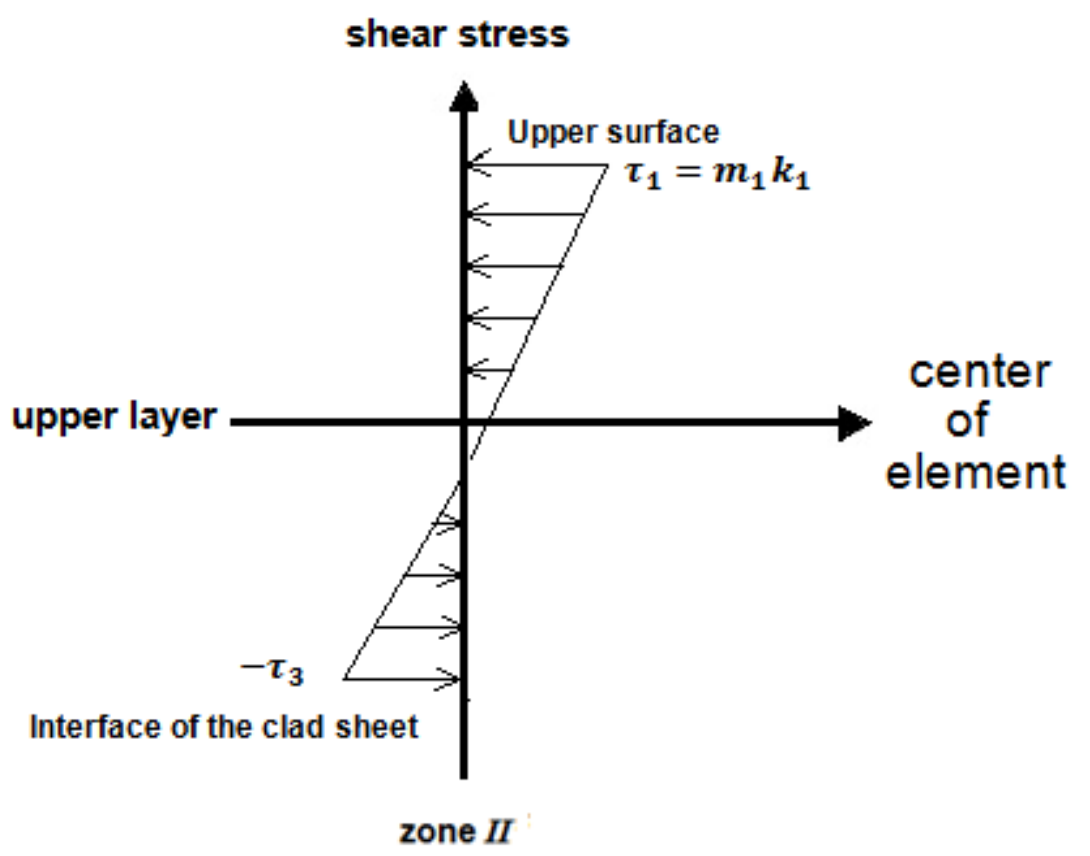

(a) 


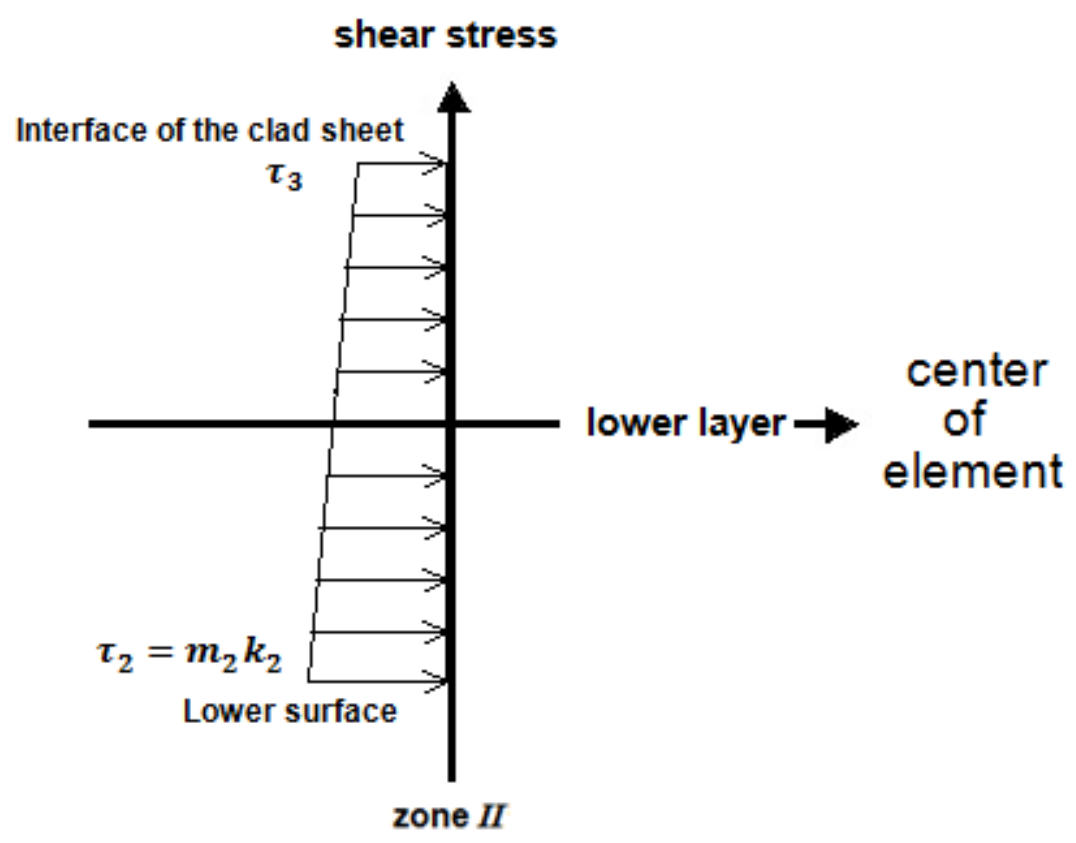

(b)

Fig.4. Distribution the shear stress on the vertical elements of zone II for the (a) upper layer (b) lower layer

Moreover, sheets have been bonded completely in this zone, but the thickness ratios of clad sheet at the entry and exit are dissimilar, namely $\beta_{i}=\frac{h_{i 1}}{h_{1}}$ and $\beta_{o}=\frac{h_{b 1}}{h_{b}}=\frac{h_{o 1}}{h_{o}}$, respectively.

According to the stress field of Fig. 2 (b), the horizontal and vertical force equilibriums for layers results to the following relations, respectively

$$
\begin{aligned}
& \frac{d\left(h_{1} \sigma_{1}\right)}{d x}+\left(\tan \theta_{1}-\tan \theta_{3}\right) p-\left(\tan ^{2} \theta_{1}+1\right) \tau_{1}-\left(\tan ^{2} \theta_{3}+1\right) \tau_{3} \\
& +\bar{\tau}_{I I 1} \frac{d\left(h_{1}\right)}{d x} \tan \theta_{1}=0 \\
& \frac{d\left(h_{2} \sigma_{2}\right)}{d x}+\left(\tan \theta_{2}+\tan \theta_{3}\right) p-\left(\tan ^{2} \theta_{2}+1\right) \tau_{2}+\left(\tan ^{2} \theta_{3}+1\right) \tau_{3}
\end{aligned}
$$


$+\bar{\tau}_{I I 2} \frac{d\left(h_{2}\right)}{d x} \tan \theta_{2}=0$

where

$\bar{\tau}_{I I 1}=\frac{1}{4}\left(\tau_{1}-\tau_{3}\right), \quad \bar{\tau}_{I I 2}=\frac{1}{2}\left(\tau_{2}+\tau_{3}\right)$

With combining Eq 16 with Eq17 and considering the geometrical conditions, the rolling pressure distribution in zoneII can be obtained as follows

$p_{I I}=-S_{2} x+H \ln \left(x^{2}+U\right)+C_{d_{2}} \omega+B_{2}^{*}$

where

$S_{2}=\left(\frac{\tau_{1}}{R_{1}^{2}}+\frac{\tau_{2}}{R_{2}^{2}}+\frac{\bar{\tau}_{I I 1}}{R_{1}{ }^{2}}+\frac{\bar{\tau}_{I I 2}}{{R_{2}}^{2}}\right) R_{e q} \quad, \quad H=2 k_{e} \quad, \quad U=R_{e q} h_{o}$

$C_{d_{2}}=\left(\frac{S_{2} U-L_{2}}{\sqrt{U}}\right), \quad L_{2}=\tau_{e 2} R_{e q} \quad, \quad \omega=\tan ^{-1} \frac{x}{\sqrt{U}}$

$k_{e}=k_{1} \beta_{o}+k_{2}\left(1-\beta_{o}\right), \quad h_{b}=h_{o}+\frac{x_{b}^{2}}{R_{e q}}, \quad h_{b 1}=h_{b}-h_{i 2}, \quad h_{o 2}=h_{o}-h_{o 1}$

At bonding point, $x_{b}$, the rolling pressures in zone IandIIare equal i.e. $p_{I}=p_{I I}$. Considering this constraint, $B_{2}^{*}$ in Eq 19 as a constant of integration can be obtained as follows

$$
\begin{aligned}
B_{2}^{*}=\left(-S_{1} x_{b}+\frac{H_{1}}{2} \ln \left(x_{b}{ }^{2}+U_{1}\right)+C_{d 1} \omega_{b}+B_{1}^{*}\right)- \\
\left(-S_{2} x_{b}+H \ln \left(x_{b}{ }^{2}+U\right)+C_{d_{2}} \omega_{b}\right)
\end{aligned}
$$

\subsubsection{Zone III $\left(\mathrm{x}_{\mathrm{n} 2} \leq \mathrm{x} \leq \mathrm{x}_{\mathrm{n} 1}\right)$}

ZoneIIIis a cross shear zone in which the clad sheet velocity is less than that of the lower roll and greater than that of the upper roll. However, the frictional stress on the upper side is backward and on the lower side is forward in zone III, i.e. $\tau_{e 3}=-\tau_{1}+\tau_{2}$. 
Furthermore, theshear stress state for a slab in zone III is illustrated in Fig. 5 in whichthe variations of the shear stress on the vertical side of the elementsfor both upper and lower layers are identical.

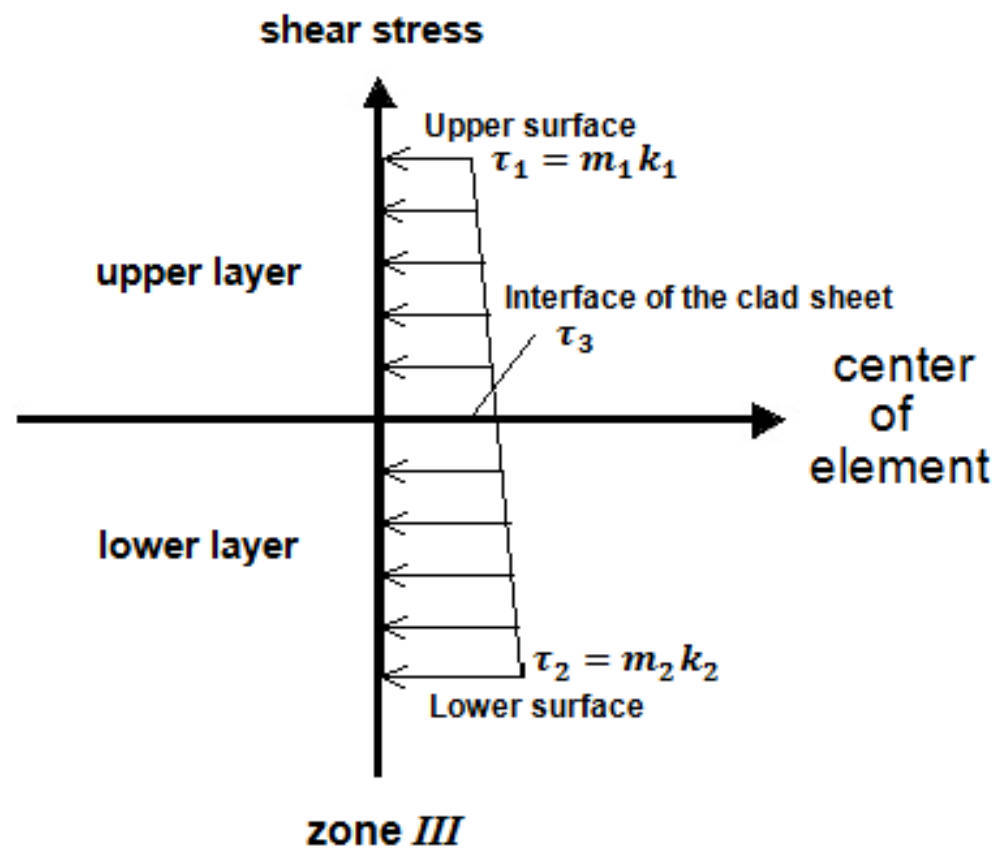

Fig.5. Shear stress distribution on the vertical element of zone III

Taking into consideration the stress field of Fig. 2 (c), and combining the horizontal and vertical force equilibriums for the element and solving the subsequent differential equation, the rolling pressure distribution in zone III can be obtained as follows

$p_{I I I}=-S_{3} x+H \ln \left(x^{2}+U\right)+C_{d_{3}} \omega+B_{3}^{*}$

where

$S_{3}=\left(-\frac{\tau_{1}}{R_{1}^{2}}+\frac{\tau_{2}}{R_{2}^{2}}+\frac{\bar{\tau}_{I I I 1}}{R_{1}{ }^{2}}+\frac{\bar{\tau}_{I I I 2}}{R_{2}{ }^{2}}\right) R_{e q}, \quad C_{d_{3}}=\left(\frac{S_{3} U-L_{3}}{\sqrt{U}}\right), \quad L_{3}=\tau_{e 3} R_{e q}$
$\bar{\tau}_{I I I 1}=\frac{1}{2}\left(\tau_{1}+\tau_{3}\right), \quad \bar{\tau}_{I I I 2}=\frac{1}{2}\left(\tau_{2}+\tau_{3}\right)$ 
In the upper neutral point $\left(x=x_{n 1}\right)$, there are the same rolling pressures in zone IIandIII, $\left(p_{I I}=p_{I I I}\right)$. Consequently, the constant of integration $\left(B_{3}^{*}\right)$ can be obtained as follows

$B_{3}^{*}=\left(-S_{2} x_{n 1}+C_{d_{2}} \omega_{n 1}+B_{2}^{*}\right)-\left(-S_{3} x_{n 1}+C_{d_{3}} \omega_{n 1}\right)$

where

$\omega_{n 1}=\tan ^{-1} \frac{x_{n 1}}{\sqrt{U}}$

\subsubsection{Zone $I V\left(0 \leq \mathrm{x} \leq \mathrm{x}_{\mathrm{n} 2}\right)$}

In zoneIV, the velocity of the clad sheet is higher than that of the upper and lower rolls; hence the frictional stresses on both side of the clad sheet are backward i.e. $\tau_{e 4}=$ $-\left(\tau_{1}+\tau_{2}\right)$. The shear stress distributions on the vertical elementin zone $I V$ are shown in Fig.6.

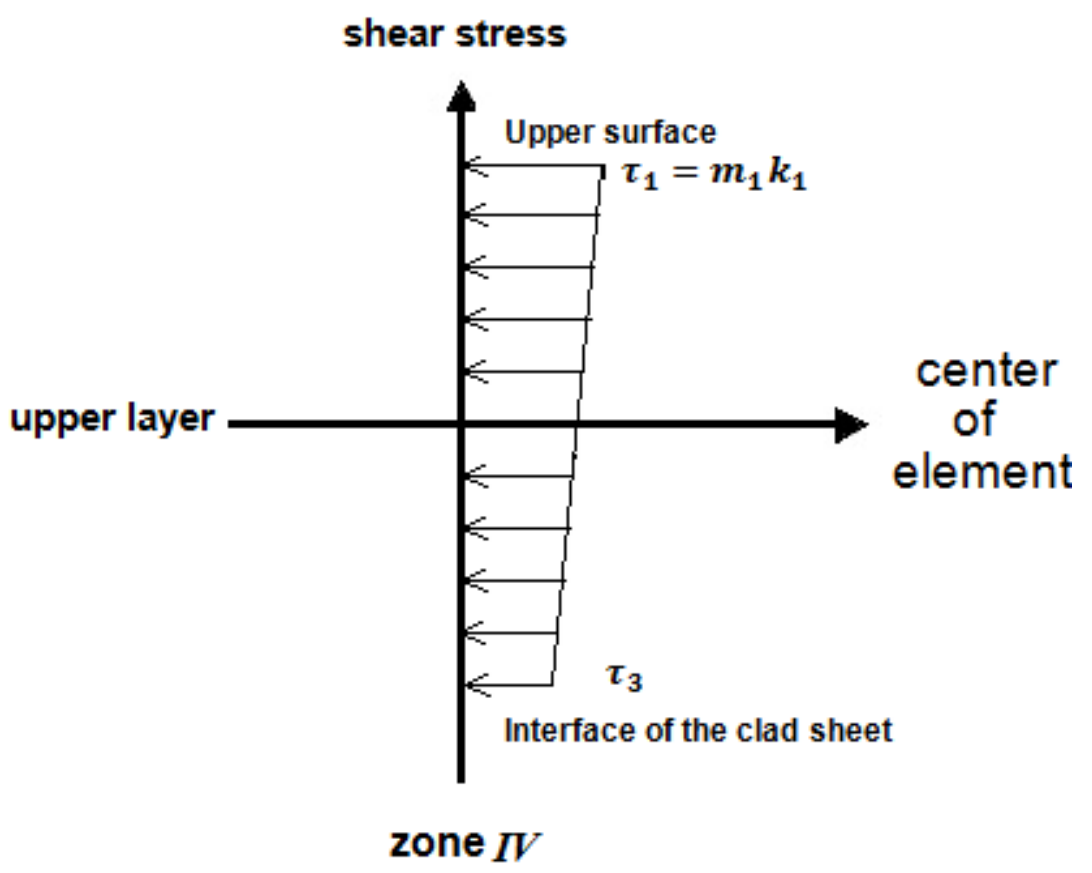

(a) 


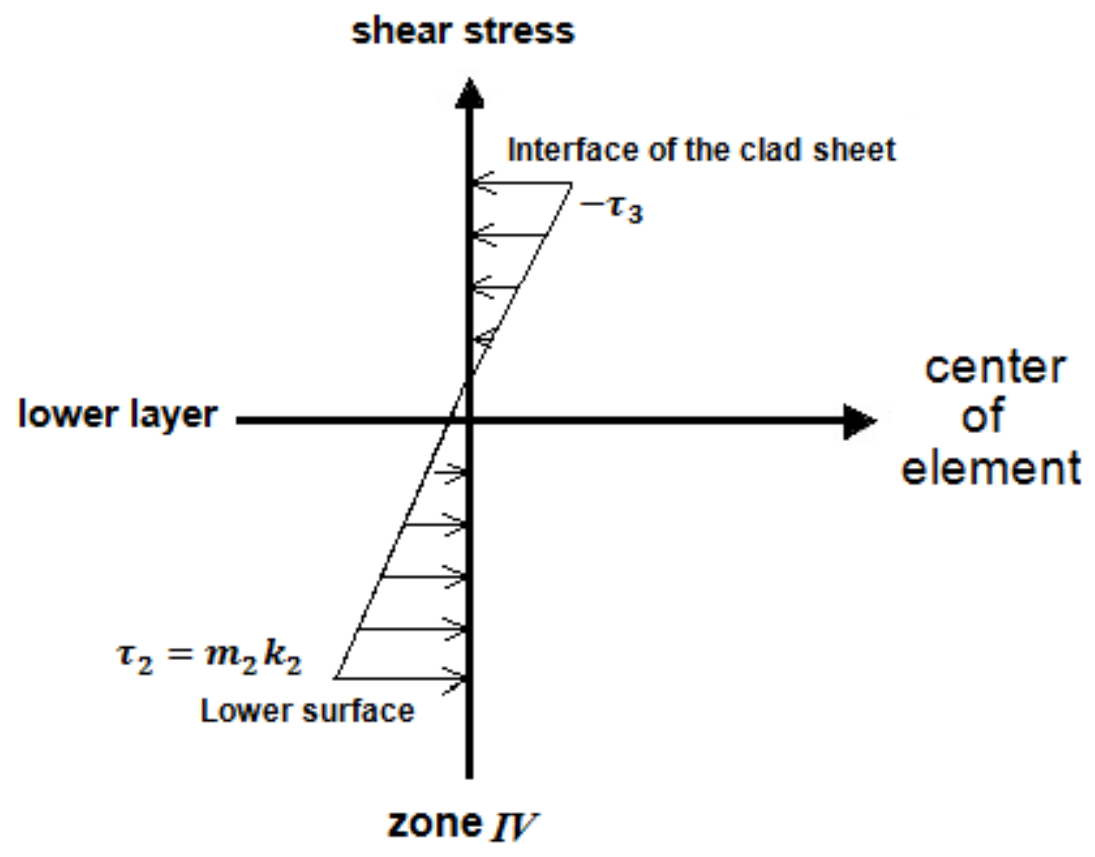

(b)

Fig.6. Distribution the shear stress on the vertical element of zone IVfor the (a) upper layer (b) lower layer

Based on the stress field of Fig. 2 (d), using the horizontal and vertical force equilibriums and solving the consequent differential equation, the rolling pressure distribution in zone $I V$ can be obtained as follows

$p_{I V}=-S_{4} x+H \ln \left(x^{2}+U\right)+C_{d_{4}} \omega+B_{4}^{*}$

where

$S_{4}=\left(-\frac{\tau_{1}}{R_{1}^{2}}-\frac{\tau_{2}}{R_{2}^{2}}+\frac{\bar{\tau}_{V 1}}{R_{1}{ }^{2}}+\frac{\bar{\tau}_{T V 2}}{R_{2}{ }^{2}}\right) R_{e q} \quad, \quad C_{d_{4}}=\left(\frac{S_{4} U-L_{4}}{\sqrt{U}}\right) \quad, \quad L_{4}=\tau_{e 4} R_{e q}$

$\bar{\tau}_{I V 1}=\frac{1}{2}\left(\tau_{1}+\tau_{3}\right), \quad \bar{\tau}_{I V 2}=\frac{1}{4}\left(\tau_{2}-\tau_{3}\right)$

The boundary condition at the exit is

$$
\sigma=\sigma_{\mathrm{o}} \quad \text { or } p_{o}=2 k_{e}-\sigma_{\mathrm{o}} \quad \text { at } \quad x=0(\text { or } \omega=0)
$$


Applying the Eq28 on to Eq27, the constant $\mathrm{B}_{4}^{*}$ can be obtained as follows

$B_{4}^{*}=2 k_{e}(1-\ln U)-\sigma_{\mathrm{o}}$

$p_{I}, p_{I I}, p_{I I I}$ and $p_{I V}$ are the roll pressures at the zones $I, I I, I I I$ and $I V$, respectively. The specific shear stresses in four zones can be obtainedas follows

$\left(\tau_{3}\right)_{I}=m_{3} k_{1}$ zone $I$

$\left(\tau_{3}\right)_{I I}=\frac{\varphi_{22}-\gamma_{2}+\tau_{1} \varnothing \varphi_{2}+2 \tau_{2} \varnothing \varphi_{1}}{\alpha_{2}-\varphi_{2} \varnothing-2 \varphi_{1} \varnothing}$ zone $I I$

$\left(\tau_{3}\right)_{I I I}=\frac{\varphi_{32}-\gamma_{2}+2 \tau_{1} \varnothing \varphi_{2}+2 \tau_{2} \varnothing \varphi_{1}}{\alpha_{2}-2 \varphi_{2} \emptyset-2 \varphi_{1} \varnothing}$ zone $I I I$

$\left(\tau_{3}\right)_{I V}=\frac{\varphi_{42}-\gamma_{2}+2 \tau_{1} \varnothing \varphi_{2}-\tau_{2} \emptyset \varphi_{1}}{\alpha_{2}-\varphi_{2} \varnothing-2 \varphi_{1} \varnothing}$ zone $I V$

where

$\varphi_{1}=\frac{2 \beta_{o} x}{R_{e q}}, \quad \varphi_{2}=\frac{2\left(1-\beta_{o}\right) x}{R_{e q}} \quad, \quad \varnothing=\frac{x^{2}}{4 R_{1}^{2}}$

$\varphi_{22}=m_{2} k_{2}\left(\frac{x^{2}}{R_{2}^{2}}+1\right) \varphi_{1}-m_{1} k_{1}\left(\frac{x^{2}}{R_{1}^{2}}+1\right) \varphi_{2}$

$\gamma_{2}=\frac{4 \beta_{o}\left(1-\beta_{o}\right) x^{2}}{R_{e q}{ }^{2}}\left(2 k_{2}-2 k_{1}\right), \quad \alpha_{2}=\frac{2 x}{R_{e q}}\left(\left(\frac{1}{R_{1}}-\frac{2 \beta_{o}}{R_{e q}}\right)^{2} x^{2}+1\right)$

$\varphi_{32}=-\left(m_{2} k_{2}\left(\frac{x^{2}}{R_{2}^{2}}+1\right) \varphi_{1}+m_{1} k_{1}\left(\frac{x^{2}}{R_{1}^{2}}+1\right) \varphi_{2}\right)$

$\varphi_{42}=-m_{2} k_{2}\left(\frac{x^{2}}{R_{2}^{2}}+1\right) \varphi_{1}+m_{1} k_{1}\left(\frac{x^{2}}{R_{1}^{2}}+1\right) \varphi_{2}$

\subsection{Determination of the neutral points}

Position of the neutral points, $x_{n 1}$ and $x_{n 2}$ can be found by using the followingconstraints 
At $x=x_{n 1}$, the rolling pressure in zoneII andIIIare equal, i.e., $p_{I I}=p_{I I I}$

$-S_{2} x+H \ln \left(x^{2}+U\right)+C_{d_{2}} \omega+B_{2}^{*}=-S_{3} x+H \ln \left(x^{2}+U\right)+C_{d_{3}} \omega+B_{3}^{*}$

Moreover, at $x=x_{n 2}$, the rolling pressure in zone III and $I V$ are equal, i.e., $p_{I I I}=p_{\mathrm{IV}}$

$-S_{3} x+H \ln \left(x^{2}+U\right)+C_{d_{3}} \omega+B_{3}^{*}=-S_{4} x+H \ln \left(x^{2}+U\right)+C_{d_{4}} \omega+B_{4}^{*}$

Combining Eqs. (32) and (33), the following relation is obtained

$\left(S_{3}-S_{2}\right) \mathrm{x}_{\mathrm{n} 1}+\left(C_{d_{2}}-C_{d_{3}}\right) \omega_{n 1}+B_{2}^{*}-\left(S_{3}-S_{4}\right) \mathrm{x}_{\mathrm{n} 2}-\left(C_{d_{4}}-C_{d_{3}}\right) \omega_{n 2}-B_{4}^{*}=0$

Using the volume constancy relation of the material, the positions of the upper and lower neutral points have the following relationship[16]

$x_{n 1}=\sqrt{V_{A} x_{n 2}^{2}+\left(V_{A}-1\right) \frac{h_{o}}{R_{A}}}$

where

$V_{A}=\frac{V_{2}}{V_{1}} \quad, \quad R_{A}=\frac{1}{R_{e q}}-\frac{h_{o}}{2 R_{e q}^{2}}$

Through Eqs34 and 35, positions of the neutral points $x_{n 1}$ and $x_{n 2}$ can be obtained.

\section{3 rolling force}

Since the contact angle of the rolls and that of the sheets is assumed to be small, the rolling force is given by

$F=\int_{x_{b}}^{L} p_{I} d x+\int_{x_{n 1}}^{x_{b}} p_{I I} d x+\int_{x_{n 2}}^{x_{n 1}} p_{I I I} d x+\int_{0}^{x_{n 2}} p_{I V} d x=F_{I}+F_{I I}+F_{I I I}+F_{I V}$

Where $p_{I}, p_{I I}, p_{I I I}$ and $p_{\text {IV }}$ are the roll pressures at zonesI, II, III and IV and $x_{n 1}$ and $x_{n 2}$ are the upper and lower neutral points, respectively. 


\section{4 rolling torque}

The rolling torque per unit width can be calculated by integrating the moment of the frictional shear force along the arc length of contact around the roll axis. Therefore, the rolling torque for the upper roll can be expressed as

$$
\begin{gathered}
T_{1}=R_{1}\left(+\int_{x_{b}}^{L} m_{1} k_{1} d x+\int_{x_{n 1}}^{x_{b}} m_{1} k_{1} d x-\int_{x_{n 2}}^{x_{n 1}} m_{1} k_{1} d x-\int_{0}^{x_{n 2}} m_{1} k_{1} d x\right) \\
=R_{1} m_{1} k_{1}\left(L-2 x_{n 1}\right)
\end{gathered}
$$

and the rolling torque for the lower roll can be expressed as

$$
\begin{gathered}
T_{2}=R_{2}\left(+\int_{x_{b}}^{L} \mathrm{~m}_{2} \mathrm{k}_{2} d x+\int_{x_{n 1}}^{x_{b}} \mathrm{~m}_{2} \mathrm{k}_{2} d x+\int_{x_{n 2}}^{x_{n 1}} \mathrm{~m}_{2} \mathrm{k}_{2} d x-\int_{0}^{x_{n 2}} \mathrm{~m}_{2} \mathrm{k}_{2} d x\right) \\
=R_{2} \mathrm{~m}_{2} \mathrm{k}_{2}\left(L-2 x_{n 2}\right)
\end{gathered}
$$

Therefore, total rolling torque can be obtained as follows

$T=T_{1}+T_{2}$

\section{Finite element Simulation}

To verify the analytical model, finite element simulation of asymmetrical clad sheet unbonded before rolling was carried out using ABAQUS/explicit software. Due to the asymmetrical nature of the clad sheet rolling process, the size and/orrotational speeds of both rolls may be different.Moreover, with assuming plane strain condition, the process is simulated in two dimensions. The rolls are modeled as discrete rigid bodies and the sheets are assumed as elastic perfect plastic materials.

Aluminum as material of the upper layer and copper as material of the lower layer with the material properties given in Table 1 were considered in the simulation. Shear yield 
stress of the material was also calculated based on the Von Mises yield criterion, i.e. $\mathrm{k}=0.577 \sigma_{\mathrm{y}}$.

Table.1 Material properties of aluminum and copper in the FE simulation

\begin{tabular}{|c|c|c|c|c|}
\hline Layer & Materials & $\begin{array}{c}\text { poisson's ratio } \\
v\end{array}$ & $\begin{array}{l}\text { Modulus of } \\
\text { Elasticity } \\
\text { E (GPa) }\end{array}$ & $\begin{array}{l}\text { Density } \\
\rho\left(\mathrm{kg} / \mathrm{m}^{3}\right)\end{array}$ \\
\hline Upper & $\mathrm{Al}$ & 0.36 & 68 & 2698 \\
\hline Lower & Cu 99\% & 0.343 & 110 & 7924 \\
\hline
\end{tabular}

From 2D CPE4R element type, 638 elementswith580 nodes and 1278 elements with 1116 nodes are used to mesh the upper and the lower layers, respectively.Schematic illustration of the mesh model for clad sheet in the roll gap is shown in Fig. 7. Number of elements was checked to be appropriate by applying the mesh sensitivity test.

Because of the non-linearity behavior of the process, the dynamic/explicit analysis was taken into account. The mass scaling strategy was considered to perform the convergence of the problem. Two rolls were only allowed to rotate around their centers and constrained in the other directions. There is noconstraint on the clad sheet while it is rolled between two rolls. The simulation shows that throughout the process, the thickness of the sheets are continuously reduced and their length are regularly increased. 


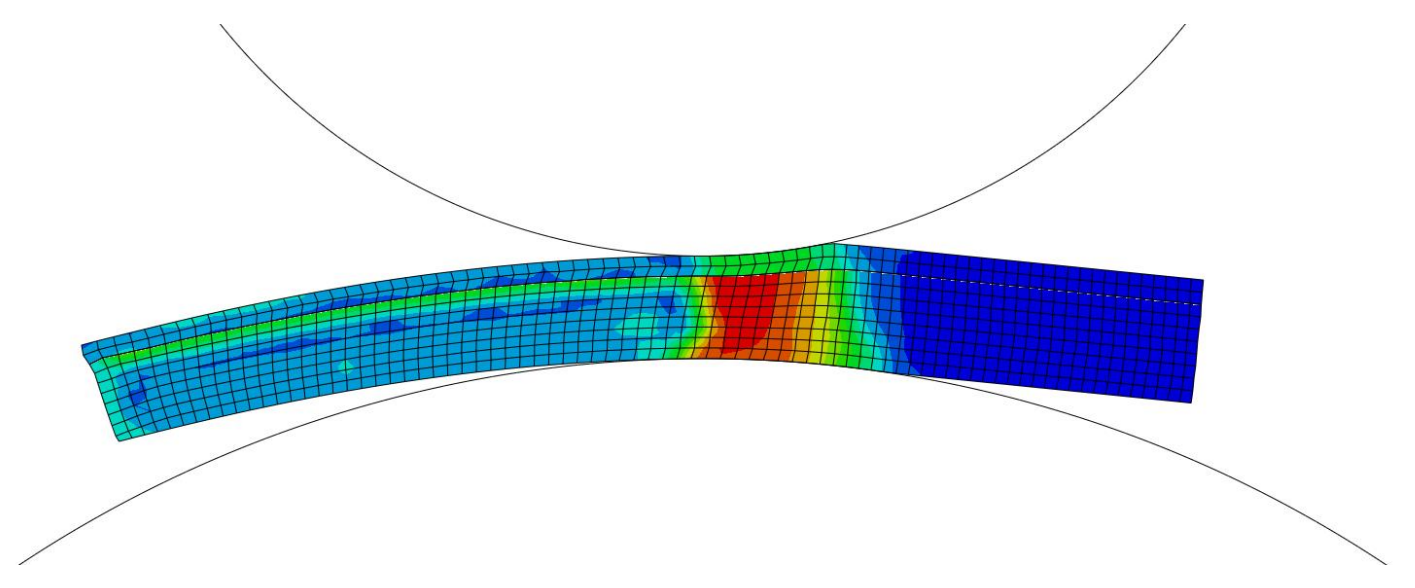

Fig. 7 Schematic illustration of the FE mesh model

\section{Results and Discussion}

From the presented mathematical model, the effective factors on the clad sheet rolling process are investigated. The effect of various shear yield stress ratios of sheets $\left(k_{2} / k_{1}\right)$ on the rolling pressure $p / 2 k_{1}$ and the rolling tensile stress $\sigma / 2 k_{1}$ distributions through the contact lengthareshown in Fig. 8.Obviously, when the shear yield stressesof both sheets are equal i.e. $k_{2}=k_{1}$, there is no bonding point.It is seen that with increasing the shear yield stress ratio, the rolling pressureand tensile stress increase as well. As the $\left(k_{2} / k_{1}\right)$ increase, the generation of bonding point is postponded and its position moves toward the output.Moreover, with increasing $\left(k_{2} / k_{1}\right)$, the magnitude of the specific shear stress at the interface of layer $\left(\tau_{3} / k_{1}\right)$ increases. Since the directions of the frictional stresses at the upper and the lower surfaces are opposite, $\tau_{3} / k_{1}$ has almost a greater magnitude in zone $I I I$. 


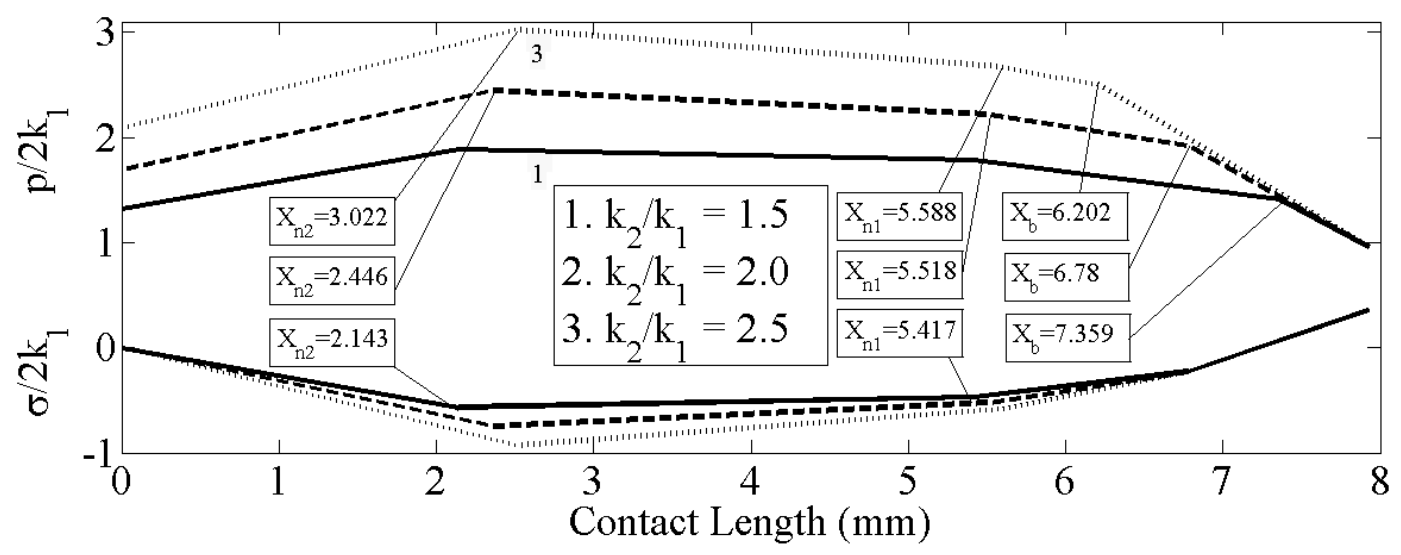

(a)

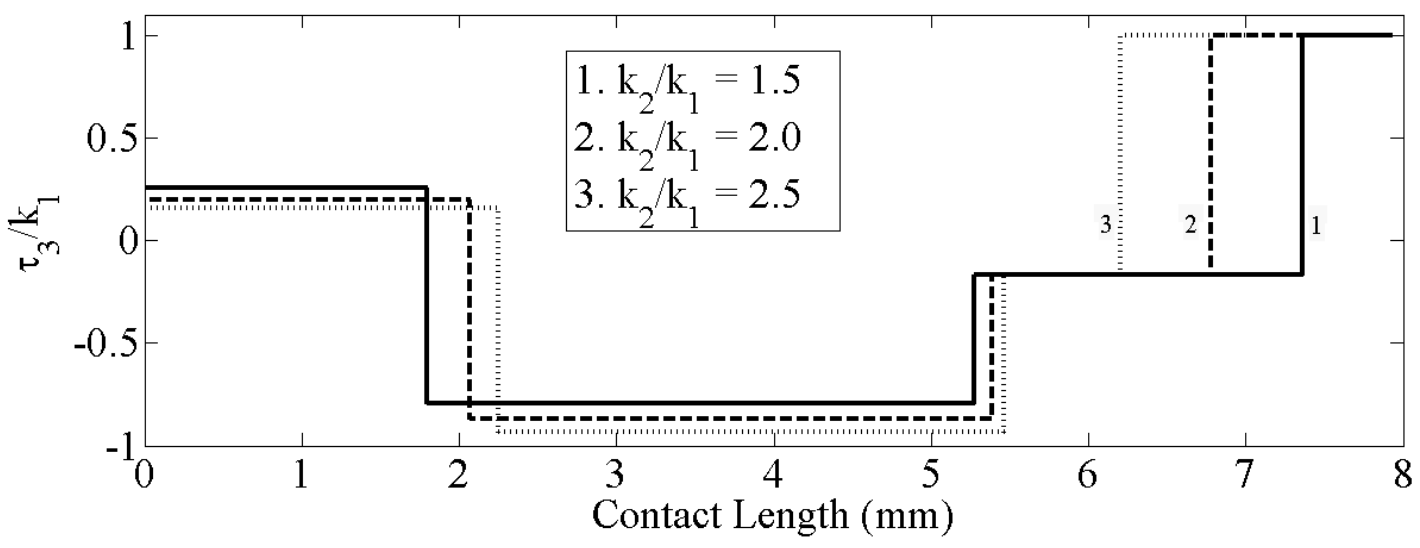

(b)

Fig.8 (a) Specific rolling pressure and the rolling tensile stress(b) the specific shear stress at the interface of layer for the various shear yield stress ratios

$$
\begin{aligned}
& R_{1}=100 \mathrm{~mm}, R_{2}=110 \mathrm{~mm}, h_{i}=3 \mathrm{~mm}, m_{1}=m_{2}=0.5, m_{3}=1 \\
& r=20 \%, V_{2} / V_{1}=1.1, \beta_{i}=0.3, \sigma_{o}=\sigma_{i 1}=\sigma_{i 2}=0, k_{1}=98.1 \mathrm{Mpa}
\end{aligned}
$$

In Fig.9, distributions of rolling pressure $p / 2 k_{1}$ and rolling tensile stress $\sigma / 2 k_{1}$ along the contact length with respect to the various friction factors ratios $m_{2} / m_{1}\left(m_{1}\right.$ is fixed) are observed. It is seen that $p / 2 k_{1}$ and $\sigma / 2 k_{1}$ increase with increasing the friction factors ratios. When $m_{2} / m_{1}=1$ and 1.5 , the specific rolling pressureat the lower neutral point is 
larger than that one for the upper neutral point.But for $m_{2} / m_{1}=0.5$, different condition exists.In addition, with increasing the friction factor ratios $\left(m_{2} / m_{1}\right)$, the magnitude of the specific shear stress at the interface of layer $\tau_{3} / k_{1}$ increases. Moreover, $\tau_{3} / k_{1}$ has the greatest magnitude in zone $I I I$.

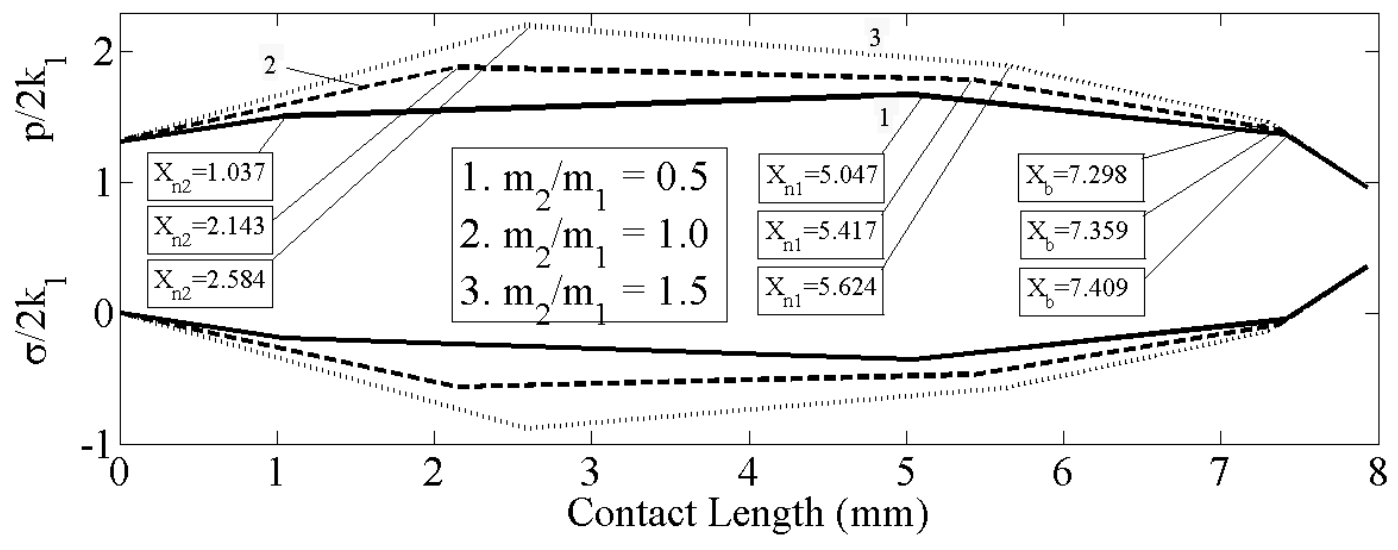

(a)

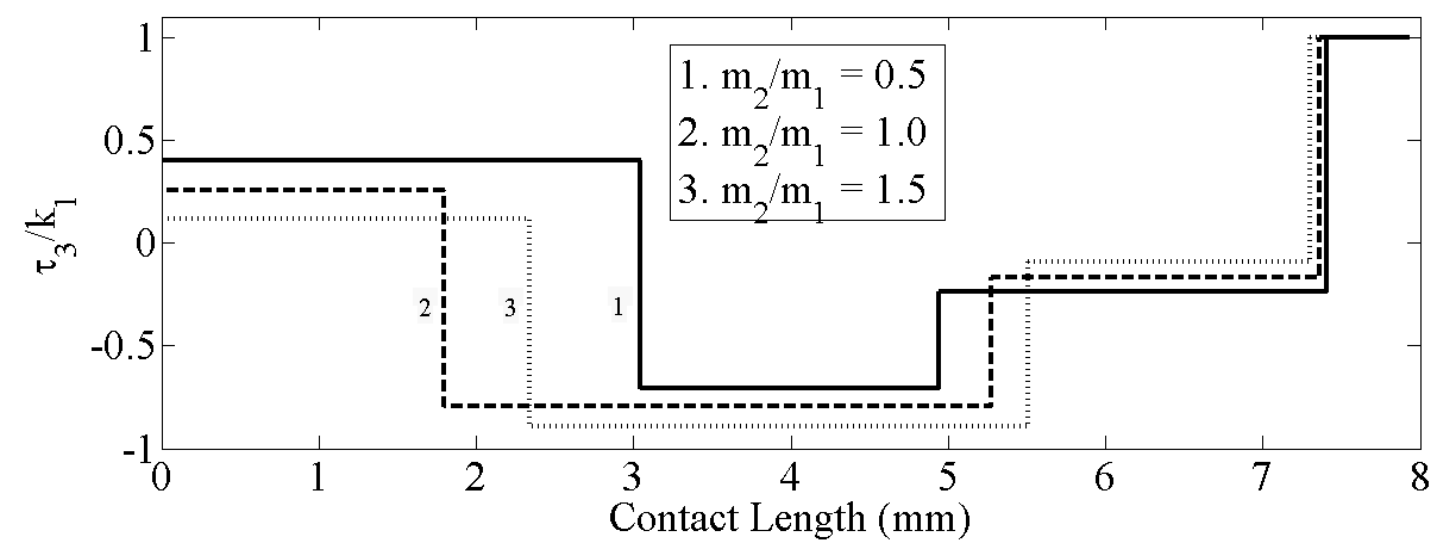

(b)

Fig.9 (a) Specific rolling pressure and the rolling tensile stress (b) the specific shear stress at the interface of layer for the various friction factors ratios

$$
\begin{gathered}
R_{1}=100 \mathrm{~mm}, R_{2}=110 \mathrm{~mm}, h_{i}=3 \mathrm{~mm}, m_{1}=0.5, m_{3}=1, k_{1}=98.1 \mathrm{Mpa} \\
r=20 \%, k_{2} / k_{1}=1.5, V_{2} / V_{1}=1.1, \beta_{i}=0.3, \sigma_{o}=\sigma_{i 1}=\sigma_{i 2}=0
\end{gathered}
$$


Fig. 10shows the effect of the various speeds ratios $\left(V_{2} / V_{1}\right)$ on the rolling pressure $p /$ $2 K_{1}$, the rolling tensile stress $\sigma / 2 k_{1}$ and the specific shear stress at the interface of layers $\tau_{3} / k_{1}$ through the contact length. There is no cross shear zone when the rolls speeds are equal. But, thelength of cross shear zone increases with increasing the speeds ratio. Obviously, the rolling pressure increases when the speeds ratio decreases. It is obvious that the rolling pressure decreases in the asymmetrical process which confirms the advantages of this process with respect tosymmetrical rolling process.

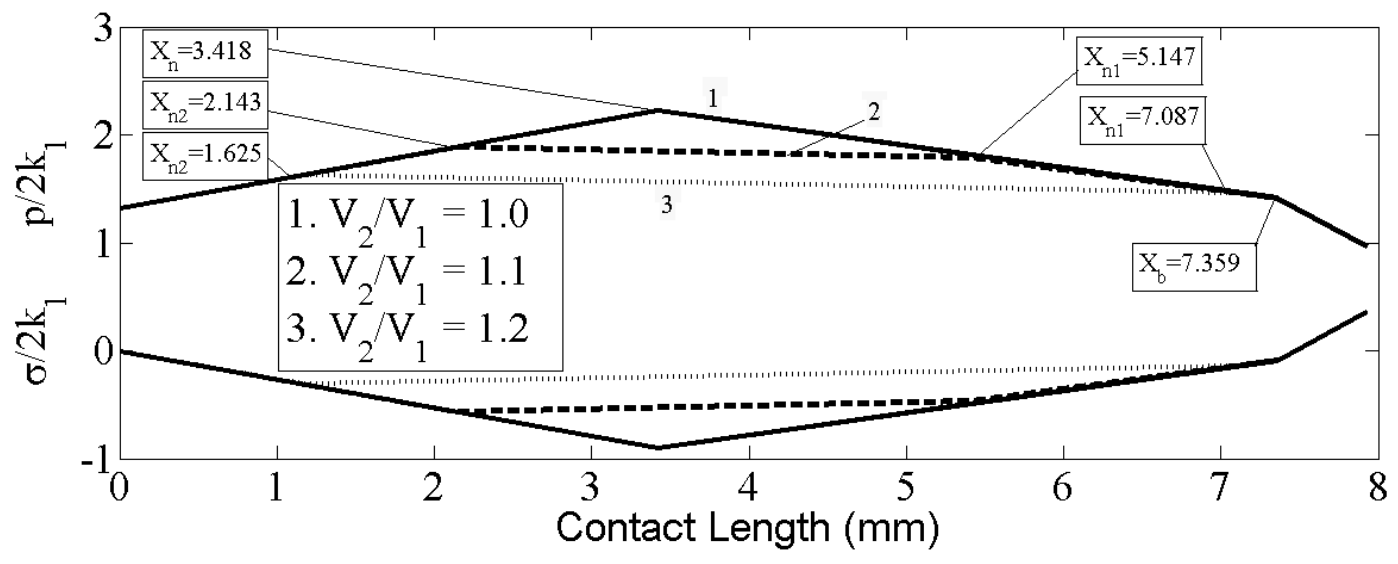

(a) 


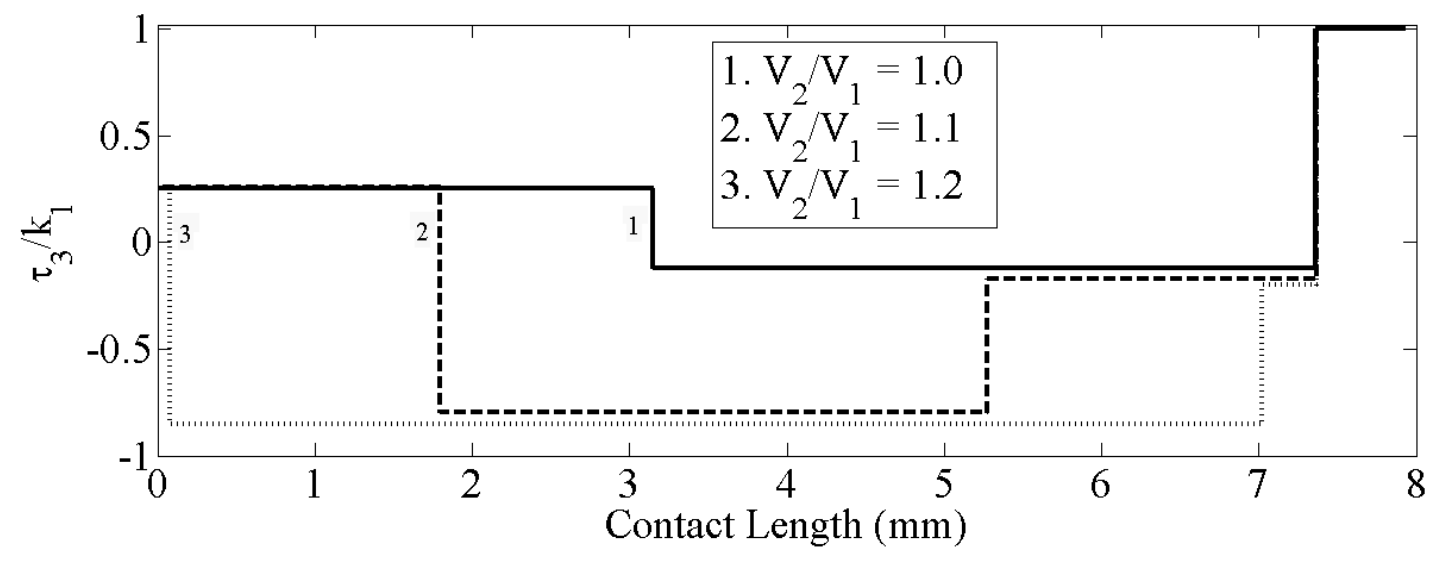

(b)

Fig.10 (a) Specific rolling pressure and the rolling tensile stress (b) the specific shear stress at the interface of layer for the various speeds ratios

$$
\begin{aligned}
& R_{1}=100 \mathrm{~mm}, R_{2}=110 \mathrm{~mm}, h_{i}=3 \mathrm{~mm}, m_{1}=m_{2}=0.5, m_{3}=1, \\
& r=20 \%, k_{2} / k_{1}=1.5, \beta_{i}=0.3, \sigma_{o}=\sigma_{i 1}=\sigma_{i 2}=0, k_{1}=98.1 \mathrm{Mpa}
\end{aligned}
$$

The effect of various roll radii ratios $R_{2} / R_{1}, R_{1}$ is assumed to be fixed, on rolling pressure $p / 2 k_{1}$ and tensile stress $\sigma / 2 k_{1}$ along the contact length are shown in Fig. 11 . Clearly, as $R_{2} / R_{1}$ increases, $p / 2 k_{1}$ and $\sigma / 2 k_{1}$ increase too. In addition, the contact length as well as the distance between the neutral points increase i.e. thelength of cross shear zone increases. Instead, increasing the roll radii ratio has no influence on the specific shear stress at the interface of the layers. 


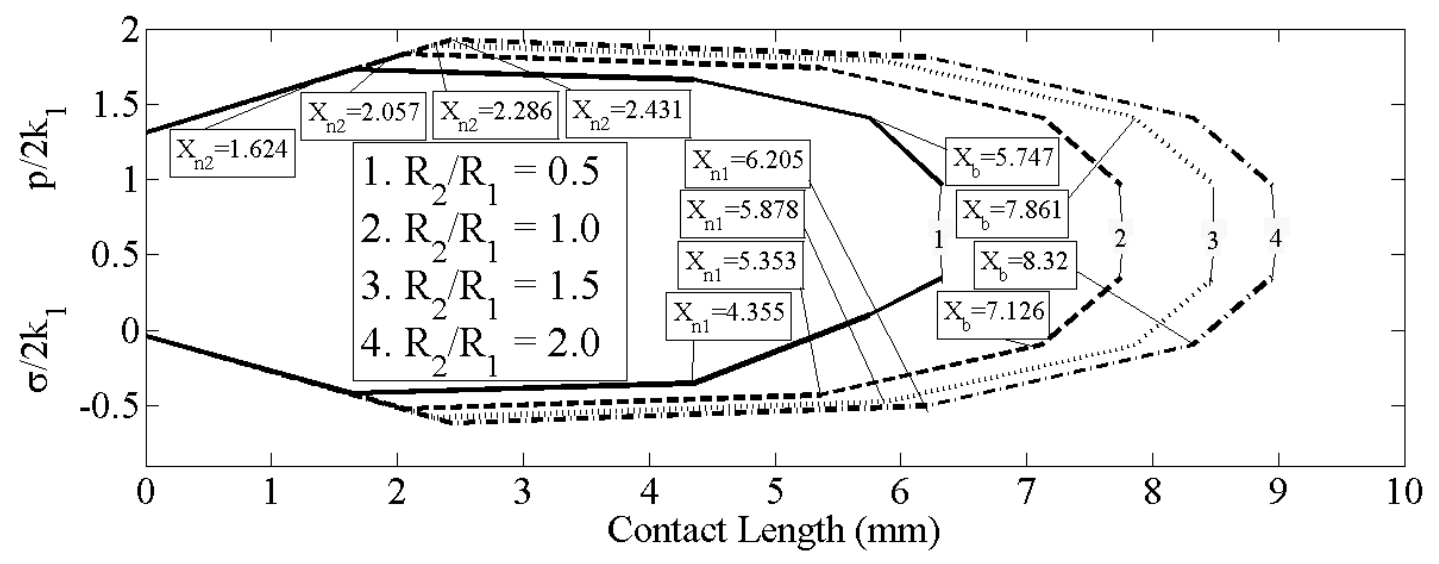

(a)

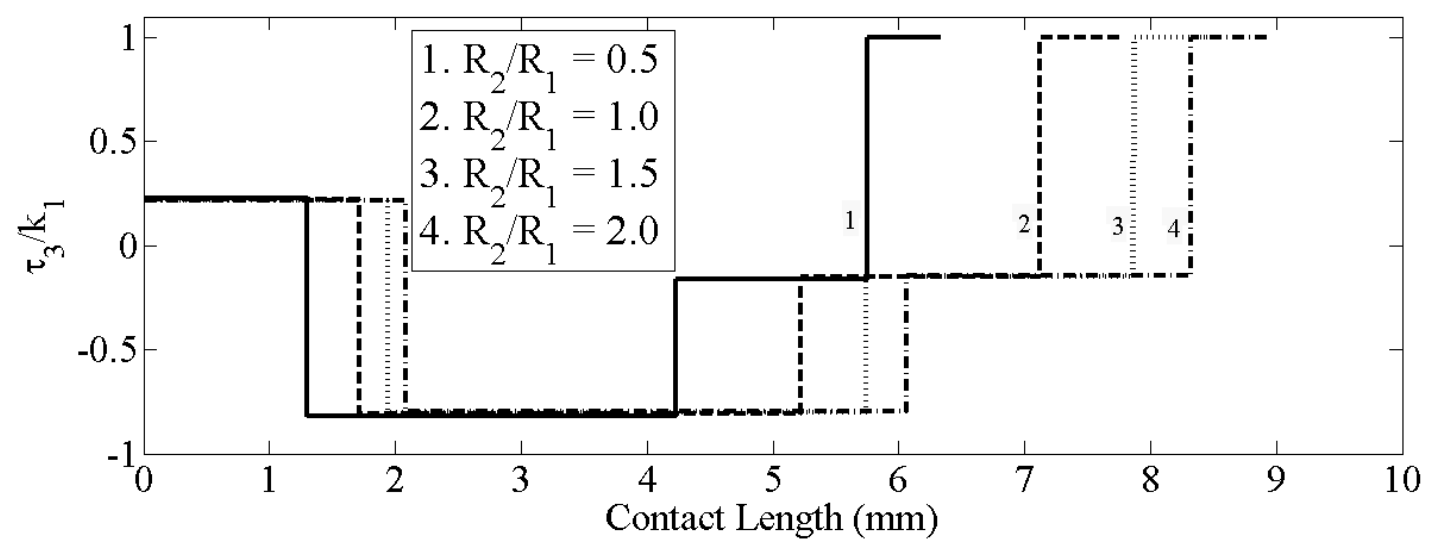

(b)

Fig.11 (a) Specific rolling pressure and the rolling tensile stress (b) the specific shear stress at the interface of layer for the various speeds ratios

$$
\begin{gathered}
R_{1}=100 \mathrm{~mm}, h_{i}=3 \mathrm{~mm}, m_{1}=m_{2}=0.5, m_{3}=1, k_{1}=98.1 \mathrm{Mpa} \\
r=20 \%, k_{2} / k_{1}=1.5, V_{2} / V_{1}=1.1, \beta_{i}=0.3, \sigma_{o}=\sigma_{i 1}=\sigma_{i 2}=0
\end{gathered}
$$

Fig. 12 shows the effects of the roll radius ratio $\left(R_{2} / R_{1}\right)$ on the asymmetrical clad sheet rolling force. It could be seen that the values obtained for rolling force from the present slab analysis have a fine conformity with those from the FEM results. Moreover, it is seen as $R_{2} / R_{1}$ increases, the total rolling force increases too. 


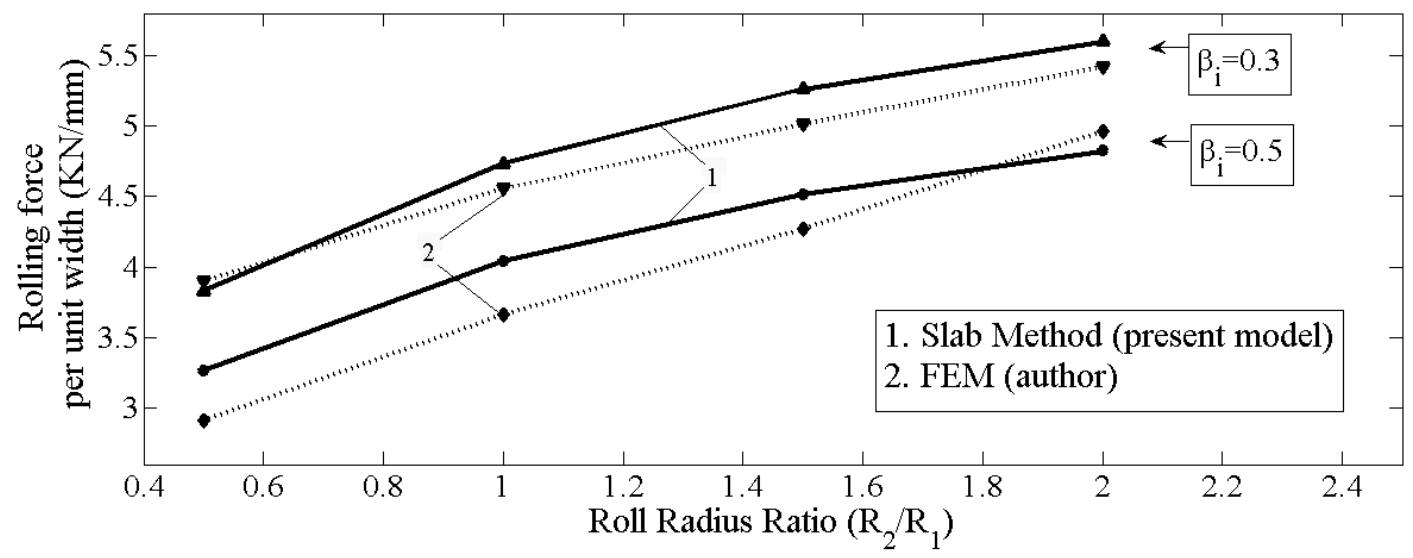

Fig. 12Asymmetrical clad sheet rolling force given the to the roll radius ratio $\left(R_{2} / R_{1}\right)$.

$$
\begin{aligned}
& R_{1}=100 \mathrm{~mm}, h_{i}=3 \mathrm{~mm}, m_{1}=m_{2}=0.5, m_{3}=1, k_{1}=98.1 \mathrm{Mpa} \\
& r=20 \%, k_{2} / k_{1}=1.5, V_{2} / V_{1}=1.1, \beta_{i}=0.3, \sigma_{o}=\sigma_{i 1}=\sigma_{i 2}=0
\end{aligned}
$$

The effect of the roll speed ratio $\left(V_{2} / V_{1}\right)$ on the asymmetrical clad sheet rolling force isshows in Fig. 13.Comparison between the results of thefinite element simulation with those of the present analytical model demonstrates that when the roll speed ratio $\left(V_{2} / V_{1}\right)$ increases, the rolling force decreases. In fact, having higher roll speed ratio could be considered as an advantageous of asymmetrical rolling process.

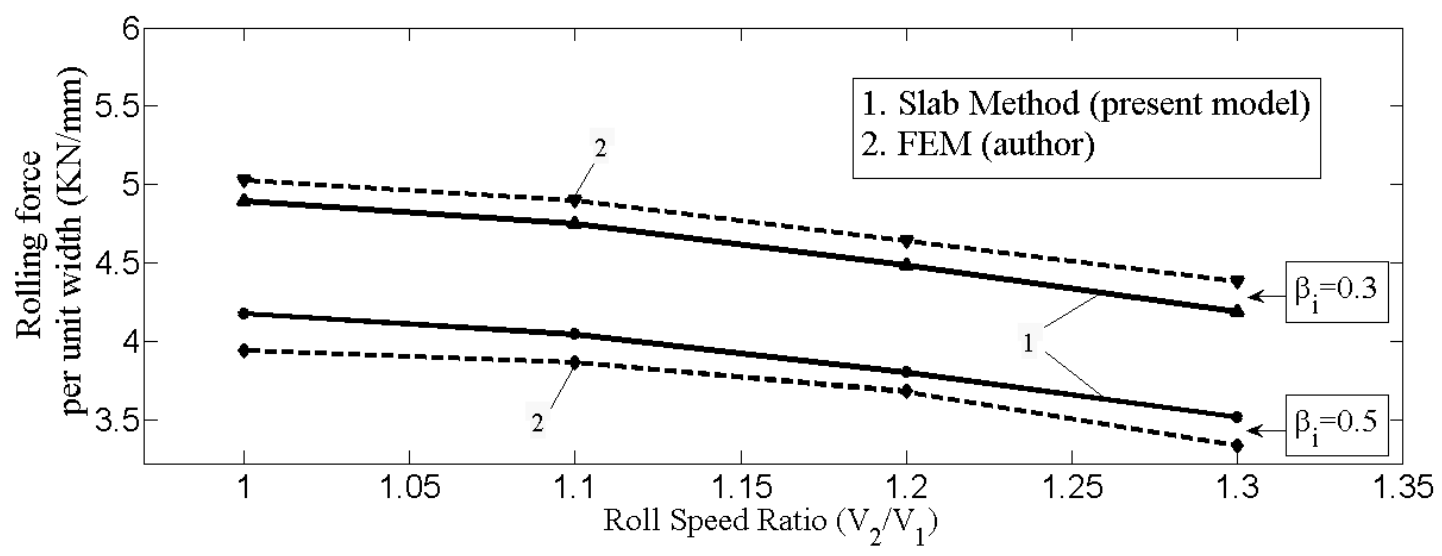


Fig. 13 Effects of the roll speed ratio $\left(V_{2} / V_{1}\right)$ on the rolling force

$$
\begin{aligned}
& R_{1}=100 \mathrm{~mm}, R_{2}=110 \mathrm{~mm}, h_{i}=3 \mathrm{~mm}, V_{1}=50 \frac{\mathrm{mm}}{\mathrm{s}}, r=20 \%, \beta_{i}=0.3 \\
& k_{1}=98.1 \mathrm{Mpa}, k_{2} / k_{1}=1.5, \sigma_{o}=\sigma_{i 1}=\sigma_{i 2}=0, m_{1}=m_{2}=0.5, m_{3}=1
\end{aligned}
$$

In Fig. 14, results of the present slab and FE analysesare compared with Pan et al. model [18] and experimental studies [1]. In the experiments of [1],Al and $\mathrm{Cu}$ were considered as materials of sheets in the asymmetrical clad sheet rolling. It should be noted that the available experimental data for asymmetric clad sheet rolling, particularlyfor the rolling force, is very inadequate and more experiments are needed. Although the results of the present model are nearly close to those from Pan model, Fig. 14 shows that the results of the present model are in a better agreement with the FE and experimental ones than those of Pan model. It should be pointed out that although the present model is less accurate than the numerical model, this is an analytical solution which can easily and quickly predict the characteristics in asymmetrical clad sheet rolling and can save computational cost. It can be seen that the rolling force for all models and analyses increases as the reductions of the clad sheet thickness increase.Some assumptions such as the real material behavior, considering the constant friction factors in the interfaces and their exact magnitudes, ignoring the roll deformation, etc. in the simulation process may be considered as reasons to have differences between the results from simulation and experimental studies in Fig. 14. 


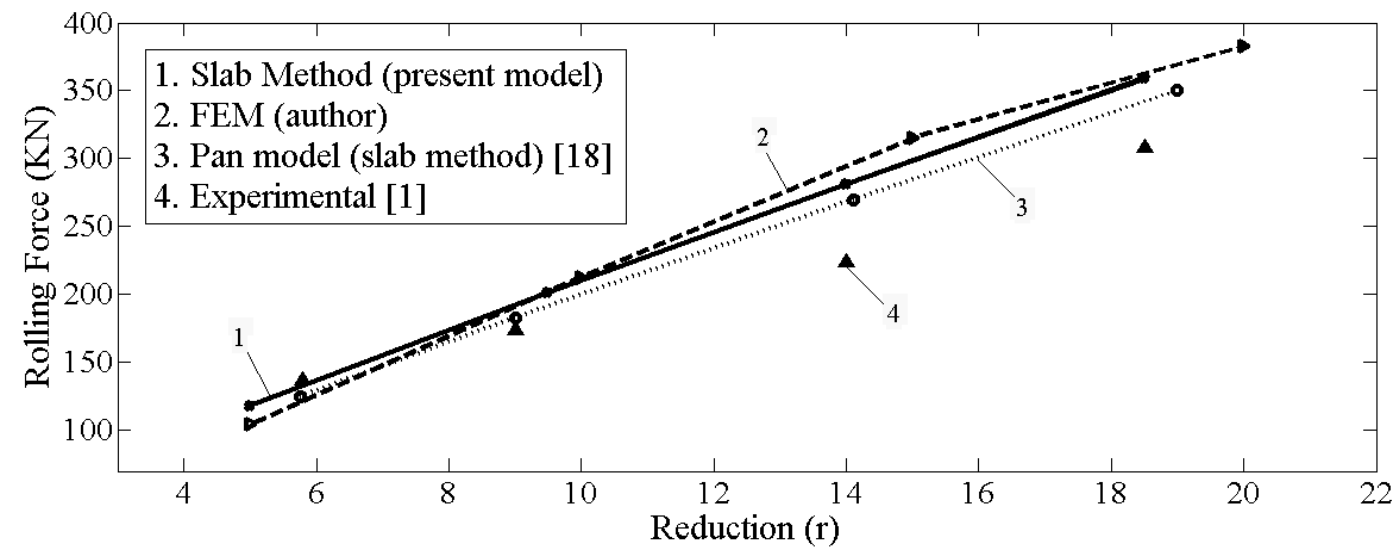

(a)

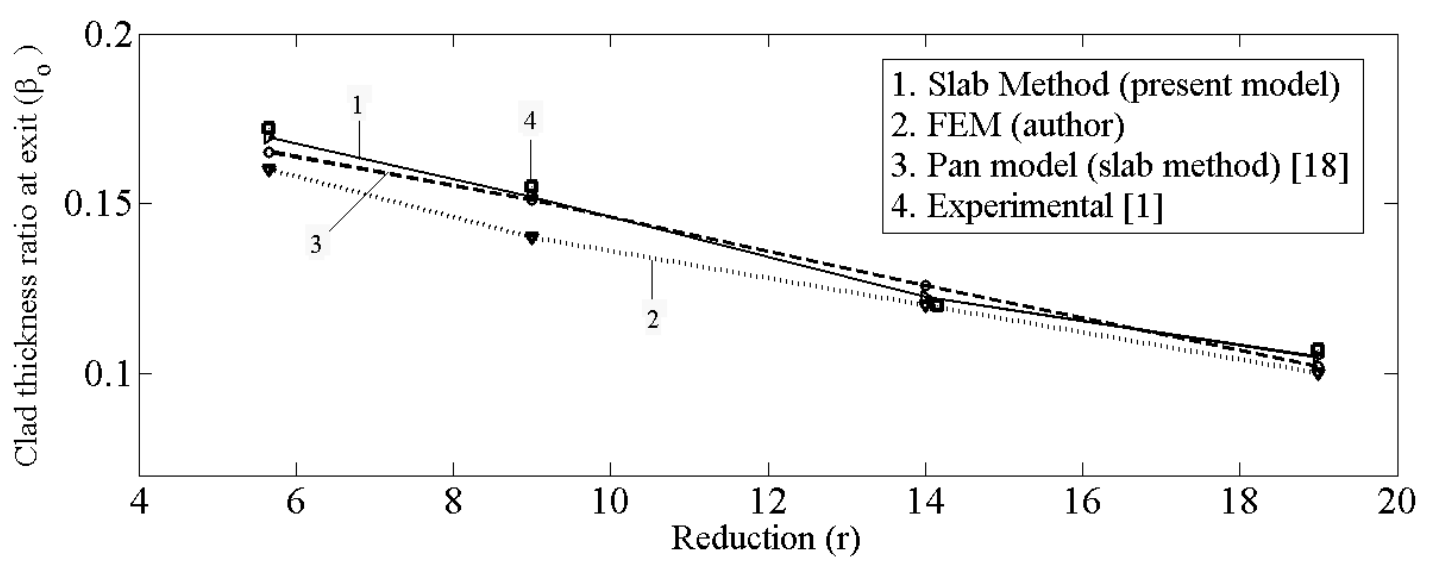

(b)

Fig. 14Comparison of (a) rolling force (b) clad thickness ratio at exit $\left(\beta_{0}\right)$ obtained from present slab and FE analyses with Pan et al. model [18] and experimental studies [1]

$$
\begin{gathered}
R_{1}=50 \mathrm{~mm}, R_{2}=100 \mathrm{~mm}, h_{i}=10 \mathrm{~mm}, V_{1}=9.44 \frac{\mathrm{mm}}{\mathrm{s}}, \frac{V_{2}}{V_{1}}=1 \\
m_{1}=m_{2}=0.5, m_{3}=1, \beta_{i}=0.2, \sigma_{o}=\sigma_{i 1}=\sigma_{i 2}=0
\end{gathered}
$$

According to the ABAQUS/explicit simulation, strain distribution in the deforming zone of asymmetrical clad sheet rolling is shown inFig. 15. In this case study, since the upper layer is softer than the lower one, the higher plastic strain happensin the upper layer which is apparent in Fig. 15. 


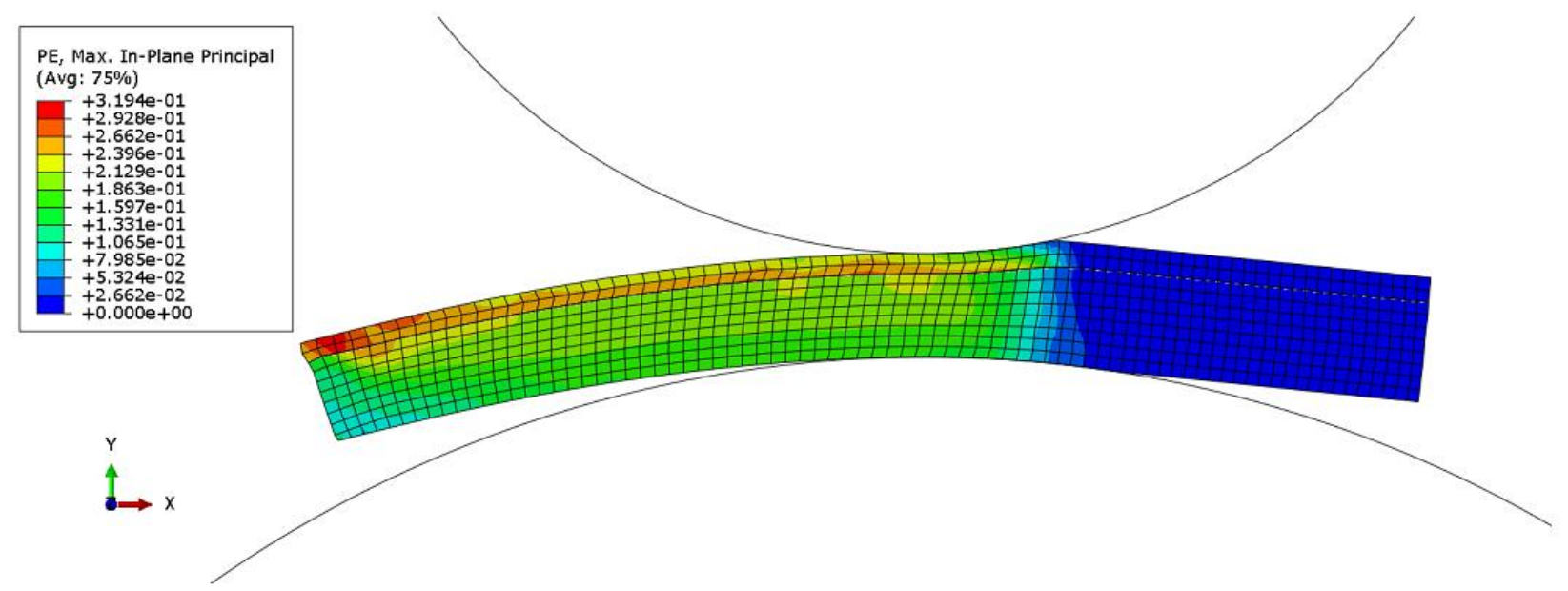

Fig. 15 Strain distributionin the deforming zone of asymmetrical clad sheet rolling

\section{Conclusions}

An analytical model for general asymmetricalclad sheet rolling is presented in which the non-uniformity of the shear stresses at the vertical sides of each slab is considered for the first time in this process. This work aims to explore the characteristics of the clad sheet unbonded before rolling by presentingthe complete expressions for the asymmetrical clad sheet rolling pressure, force and torque and predicting the position of neutral and bonding points.Moreover, by using ABAQUS/explicit software, the asymmetrical clad sheet rolling process is simulated.Using different shear yield stress ratios, roll speeds ratios, and friction factors ratios, the asymmetrical clad sheet rolling process was investigated by applying the slab method. It was found that with increasing the shear yield stress ratios or the friction factors ratios or decreasing the roll speeds ratio, the specific rolling pressure increases. It is proved that the rolling pressure in the asymmetrical clad sheet rolling process is lower than the symmetrical process which 
confirms the advantages of this process with respect to symmetrical rolling process. Moreover, as theshear yield stress ratios or the friction factors ratio decrease,the bonding point is generated sooner in deforming area.Comparison of the present slab method with FEMand experimental results, illustrate the accuracy of the present analysis.

\section{Acknowledgements}

The authors are grateful for the research support of the Iran National Science Foundation (INSF).

\section{References}

1. Hwang YM, Chen TH. Analysis of asymmetrical sheet rolling by the stream function method. JSME Int. J. Ser. A 1996;39(4):598-605.

2. Lee SH, Lee DN. Slab analysis of roll bonding of silver clad phosphor bronze sheets. Mater. Sci. Technol. 1991;7:1042-1050.

3. Johnson W. Needham G. Further experiments in asymmetrical rolling. Int. J. Mech. Sci. 1972;8:443-455.

4. Hamauzu S. The FEM analysis of hot clad sheet rolling and avoidance of curvature. Iron Steel A 1987;59:59-62.

5. Shiyouya S. The analysis of clad sheet rolling by FEM. in: Proceedings of the 40th Joint of Conference of JSTP 1989;81-84. 
6. Tzou GY. Relationship between frictional coefficient and friction factor in asymmetrical sheet rolling. J. Mater. Process. Technol. 1999;86:271-277.

7. Parvizi A, Abrinia K, Salimi M. Slab Analysis of Ring Rolling Assuming Constant Shear Friction. J. of Mate. Eng. Perform. 2011;20:1505-1511.

8. Gudur PP, Salunkhe MA, Dixit US. A theoretical study on the application of asymmetric rolling for the estimation of friction. Int. J. Mech. Sci. 2008;50:315327.

9. Yong T, Guo YH, Wang ZD, Wang GD. Analysis of Rolling Pressure in Asymmetrical Rolling Process by Slab Method. J of Iron and Steel Research, Int. 2009;16(4):22-26.

10. Tzou GY, Huang MN. Study on the minimum thickness for the asymmetrical PV cold rolling of sheet. J of Mate Process Technol. 2000;105(3):344-351.

11. Tzou GY, Huang MN. Study on minimum thickness for asymmetrical hot-and-cold PV rolling of sheet considering constant shear friction. J. Mater. Process. Technol. 2001;119(3):229-33.

12. Hwang YM, Tzou GY. An analytical approach to asymmetrical hot-sheet rolling considering the effects of the shear stress and internal moment at the roll gap. J. Mater. Process. Technol. 1995;52:399-424.

13. Gao H, Ramalingama SC, Barbera GC, Chen G. Analysis of asymmetrical cold rolling with varying coefficients of friction. J. Mater. Process. Technol. 2002;124:178-182.

14. Salimi M, Kadkhodaei M, Slab analysis of asymmetrical sheet rolling. J. Mater. Process. Technol. 2004;150:215-222. 
15. Zhang SH, Zhao DW, Gao CR, Wang GD. Analysis of asymmetrical sheet rolling by slab method. Int. J. Mech. Sci. 2012;65:168-176.

16. Hwang YM, Tzou GY. An analytical approach to asymmetrical cold- and hotrolling of clad sheet using the slab method. J. Mater. Process. Technol. 1996;62:249-259.

17. Tzou GY, Huang MN. Analytical modified model of the cold bond rolling of unbounded double-layers sheet considering hybrid friction. J. Mater. Process. Technol. 2003;140:622-627.

18. Pan SC, Huang MN, Tzou GY, Syu SW. Analysis of asymmetrical cold and hot bond rolling of unbounded clad sheet under constant shear friction. J. Mater. Process. Technol. 2006;177:114-120. 